\title{
Validation of the Decomposition Method for Fast MIMO Over-the-Air Measurements
}

\author{
Bernhard Auinger, ${ }^{1}$ Thomas Zemen, ${ }^{2}$ Michael Gadringer, ${ }^{1}$ Adam Tankielun, ${ }^{3}$ \\ Christoph Gagern, ${ }^{3}$ and Wolfgang Bösch ${ }^{1}$
}

${ }^{1}$ Graz University of Technology, Graz, Austria

${ }^{2}$ Austrian Institute of Technology, Vienna, Austria

${ }^{3}$ Rohde and Schwarz, Munich, Germany

Correspondence should be addressed to Bernhard Auinger; bernhard.auinger@tugraz.at

Received 5 October 2016; Revised 26 February 2017; Accepted 6 March 2017; Published 18 April 2017

Academic Editor: Mihajlo Stefanovic

Copyright (C) 2017 Bernhard Auinger et al. This is an open access article distributed under the Creative Commons Attribution License, which permits unrestricted use, distribution, and reproduction in any medium, provided the original work is properly cited.

Over-the-air (OTA) throughput tests of wireless Multiple-Input Multiple-Output (MIMO) devices are an important tool for network operators and manufacturers. The user equipment (UE) is placed in an anechoic chamber and a random fading process is emulated by a base-station emulator (BSE). The antenna characteristic of the UE is taken into account by sampling the sphere around the UE with the BSE test antenna at a large number of positions. For low-variance throughput results, long measurement intervals over many fading realizations are required, leading to long and expensive measurement periods in an anechoic chamber. To speed up the OTA test, we analyze the Decomposition Method (DM). The DM splits the throughput measurement into two parts: (1) a receiver algorithm performance tests taking the fading process into account and (2) an antenna performance test without fading process emulation. Both results are combined into a single throughput estimate. The DM allows for a measurement time reduction of more than one order of magnitude. We provide an analytic and numerical analysis as well as measurements. Our detailed results show the validity of the DM in all practical settings.

\section{Introduction}

With the introduction of MIMO and receiver diversity fourth- and fifth-generation $(4 \mathrm{G}, 5 \mathrm{G})$ wireless devices allow for large gains in throughput performance. These gains are highly dependent on the performance of the receive-antenna system [1] and the receiving algorithm [2] and the propagation environment [2]. The devices can change the behavior of the antenna systems, for example, by using beamforming mechanisms [3]. They also can adapt software algorithms to suit the environment they are currently used in.

Wireless equipment manufacturers as well as network providers are pushing to have performance tests of the devices available at hand. Network providers want to recommend the user equipment (UE) with the best performance to their customers; manufacturers want to be able to compare the quality of their own UE to the one of the competitor. These comparisons should include the antenna systems, the analog frontends, digital receiving algorithms, and baseband processing.

One of the methods proposed by $3 \mathrm{GPP}$, yet a very promising one-the Decomposition Method (DM) - will be investigated further in this paper. The authors' scientific contributions described in this paper are as follows:

(i) Analytical and numerical validation of the DM

(ii) Validation of the method by measurement

(iii) Detailed analysis of error sources and error bounds

Several other methods exist for performance testing of MIMO enabled UE [3-22]. A good overview is provided in [23]. Nevertheless, this paper focuses only on the DM, believing that this method can provide good and reliable results with limited effort, knowing there are limits of the method. Still, all other existing test methods also have strong sides and weaknesses too. These other performance test methods 

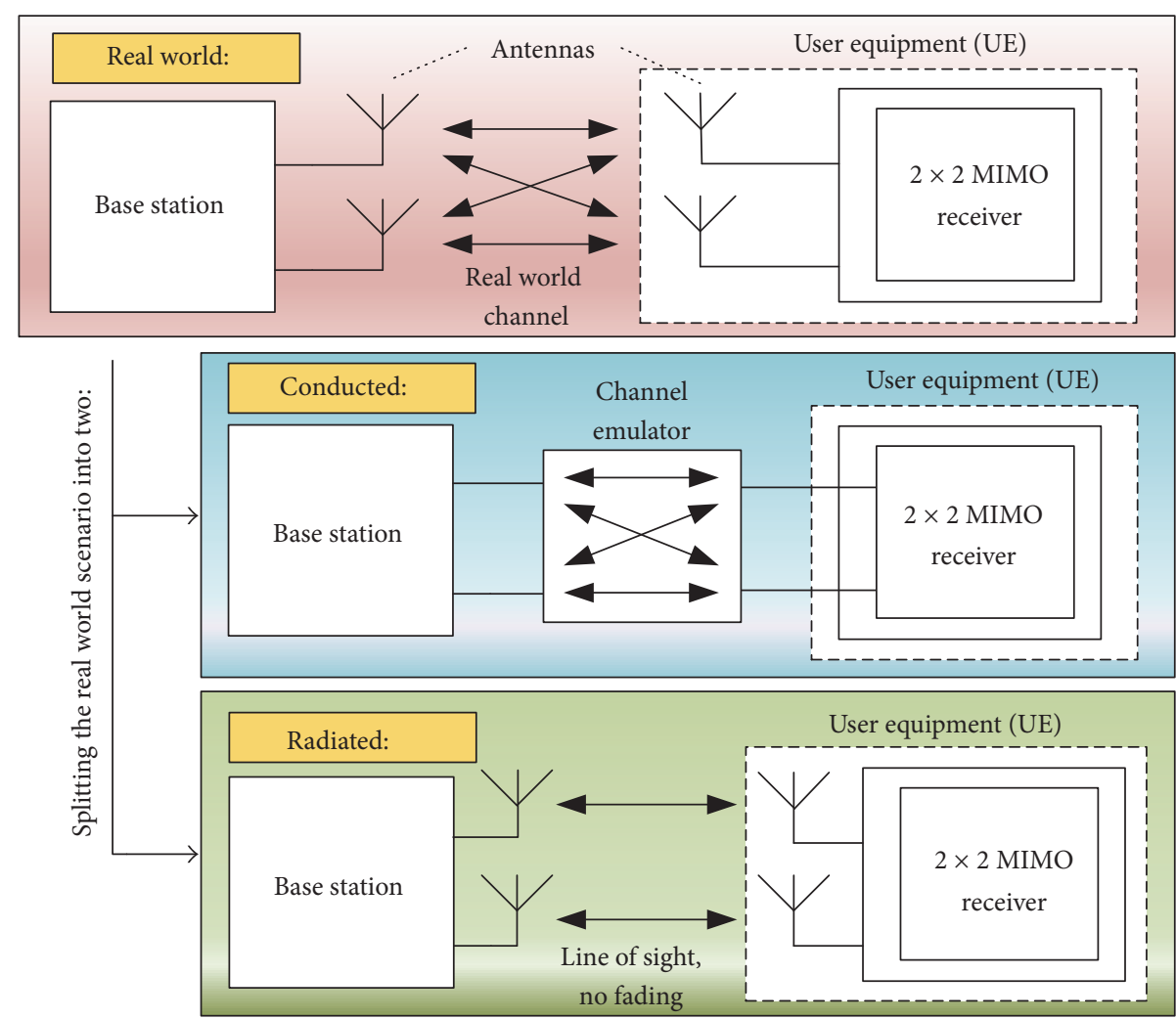

FIGURE 1: Splitting up the ideal "real world" scenario into two parts: a conducted and a radiated test setup for the downlink case of a $2 \times 2$ MIMO system [24].

will not be treated in this paper. The main advantage of the DM is that it delivers good and reliable results with limited measurement device effort and that the calibration effort of the setup is limited too.

\section{Accelerated Testing of the UE by Employing the Decomposition Method}

In a perfect test scenario, the performance of certain UE will be tested almost like it would be in the real world. The antenna performance and the algorithmic performance of the UE will be tested together in a single step (Figure 1, "real world"). The influence of the fading channel and the antennas performance are tested in one step. The test antennas have to be placed in many points to cover the whole test sphere (Figures 2 and 5(a)).

In every single point the test antennas are placed, the performance of the entire UE is tested. To get representative figures of merit of the UE performance on the propagation channel, lots of different channel realizations need to be examined. This results in a long measurement time, which is a big disadvantage in nowadays labs.

To accelerate the tests, the idea is to measure the time-consuming algorithmic performance tests only once (Figure 1, "conducted"). The antenna performance tests still have to be done in many positions, but if there is no fading channel, the wireless channel is static (Figure 1, "radiated";

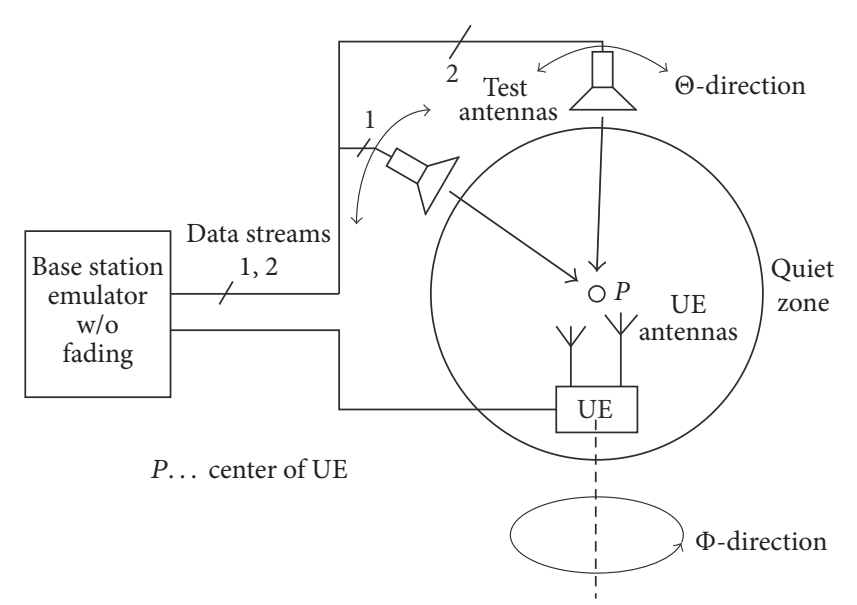

FIGURE 2: Measurement setup employing Two-Channel Method for the static radiated tests of the DM; BSE without channel emulator [25].

Figure 2) and the measurements can be done very fast, as no stochastic channel behavior has to be examined.

Later on the results of the antenna performance tests ("radiated" measurements) and the algorithmic performance tests ("conducted" measurements) are combined in a way that they are almost congruent with the "perfect" test method 
("real world" scenario), which tests all in one step. Later on the results will be combined to one single figure of merit.

This two-step approach is called Decomposition Method (DM), as it decomposes a single measurement into different single steps. The DM $[24,29]$ maps the "real world" test scenario (Figure 1) in a conducted and a radiated setup. The "real world" scenario is represented by the base station, its antenna system, the wireless propagation channel, and the UE antenna system. This scenario is split into two different measurement setups, which speeds up the measurement by my more than an order of magnitude. Both measurement results are used as a basis for calculating the throughput versus downlink power of the UE. The radiated measurements require an anechoic chamber with two moveable test antennas (Figures 2 and $5(\mathrm{a}))$.

2.1. Radiated and Conducted Setup. The conducted setup (Figure 1, "conducted") consists of the Base-Station Emulator (BSE) connected to channel emulator, which is linked to the UE by wired connections. The conducted setup is employed for two measurements:

(i) Using a fading channel ("channel" measurement): the channel emulator emulates the wireless propagation channel. It alters the signals transmitted by the BSE in a way a physical wireless propagation channel would do it, including multipath propagation (frequency dependent channels) and Doppler effects.

(ii) Using an identity matrix as channel matrix-with no fading applied ("baseline" measurement): this measurement can be seen as if the UE would directly be connected to the BSE by wires. The results of the "baseline" measurement will deliver the lowest possible downlink power values for a certain data throughput rate.

These two measurements employing the conducted setup can be done in normal laboratory environment and do not require an anechoic chamber.

The radiated setup (Figure 1, "radiated") employs the Two-Channel Method for the radiated over-the-air (OTA) tests [26]. It employs an anechoic chamber with two test antennas that are positionable in a $\Theta$-plane and a turntable for UE placement that is turnable in the $\Phi$-plane, as depicted in Figure 2. The UE is placed in the center of the anechoic chamber (quiet zone). The test antennas always have a constant distance to the UE and they are fed with signals from the BSE.

The radiated setup is static and does not contain any fading, and it is used for antenna performance tests. The two test antennas generate the EM field impinging on the UE for the radiated tests. The polarization of the test antennas can be switched independently between vertical $(\Theta)$ and horizontal $(\Phi)$ polarization. The "radiated" measurement is done with the radiated setup.

The name Two-Channel Method originates from the two downlink channels towards the UE enabled by the two test antennas (Figure 2).
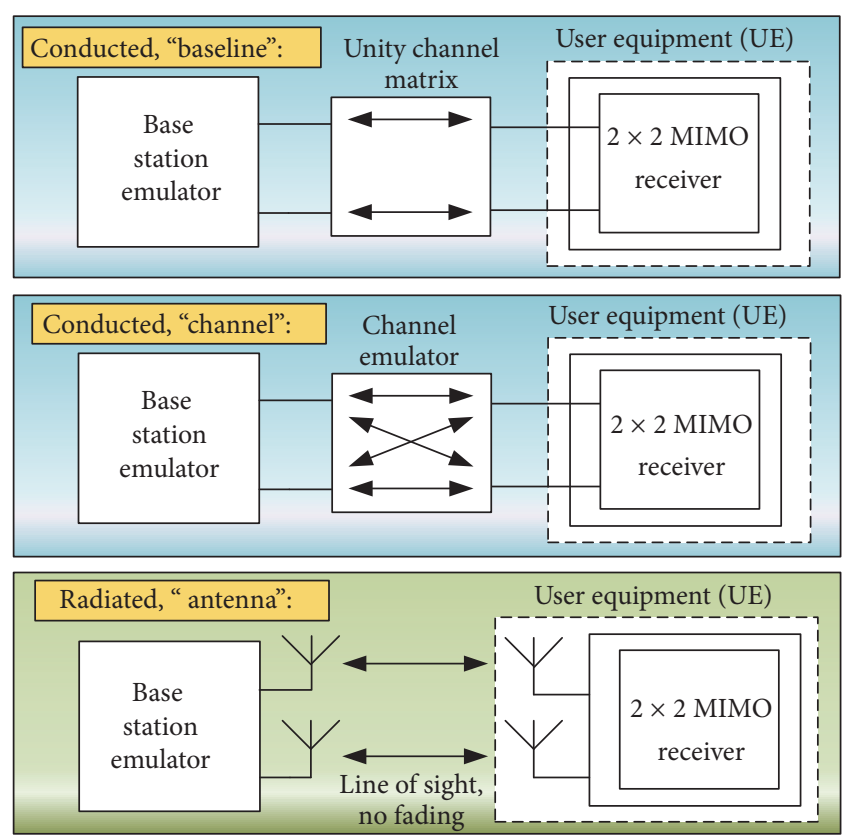

Figure 3: The split measurements: "baseline" and "channel" measurement have to be executed with the conducted setup, while "radiated" measurements employ the radiated setup.

2.2. The Split of the Measurements. The DM requires three performance test results of the UE: two using the "conducted" and one employing the "radiated" setup, as depicted in Figure 3:

(1) "Baseline" measurement: conducted measurement without fading: the user equipment is connected to the base-station emulator by two coaxial cables, the channel matrix $\mathbf{H}_{\mathrm{ch}}=\mathbf{I}$. The UE exhibits the best performance, it is only limited by the intrinsic UE noise. The result of the "baseline" measurement is the downlink power $P_{\mathrm{bl}}(y)$ required for a certain relative throughput $y$; see Figure 4 .

(2) "Channel" measurement: conducted measurement including fading: the signal is dynamically faded; Doppler shifts and spreads are applied. The ability of the device to cope with these circumstances without the UE antenna system influence is tested. The devices performance will be degraded in comparison to the "baseline" test, resulting in higher needed downlink power for a certain throughput.

The conducted faded result is $P_{\mathrm{ch}}(y)$, where the subscript ch reflects the "channel" measurement (Figure 4).

(3) "Antenna" measurement: radiated measurement without fading: the Two-Channel Method [26] is used to check the performance of the device antennas (including the receiving algorithms without its fading behavior). The radiated coupling between the test antennas and the UE is exhibited in Figure 5(a). The user has to choose a proper set of test antennas and UE positions, so-called constellations. These 


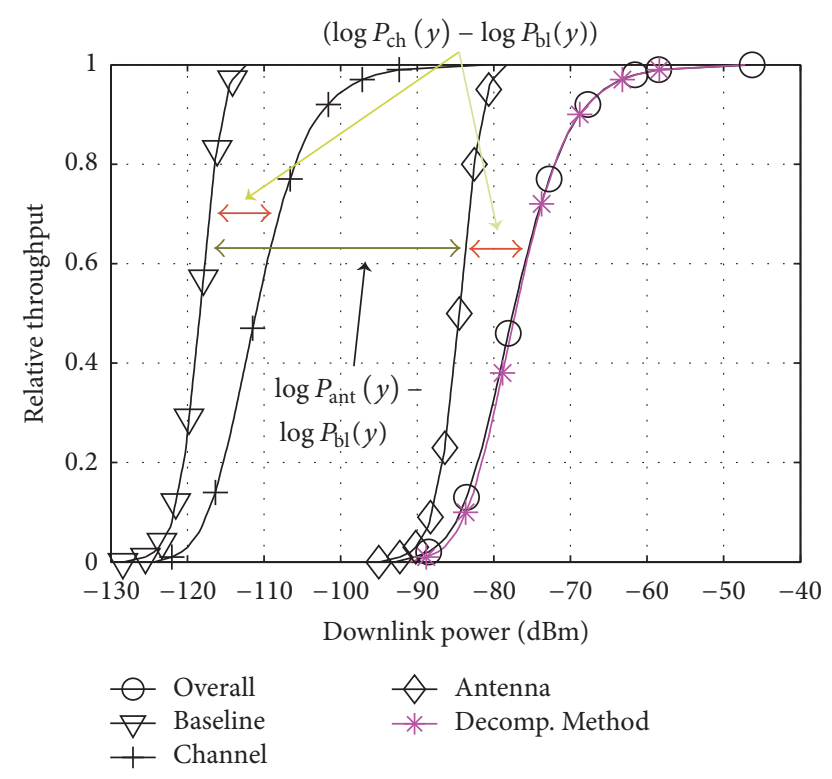

FIGURE 4: Result of the DM (relative throughput versus downlink power): adding up the logarithmic results of the "baseline"; "channel"; and "antenna" measurements. Multiply all formulas by 10 for results in $\mathrm{dB}$.

constellations also include the test antenna polarization instances, which are used during the measurements [30].

The result of the measurement is $P_{\text {ant }}(y)$, where the subscript ant reflects the "antenna" measurement (Figure 4).

2.3. Combining the DM Results to One Figure of Merit. To get a single figure of merit, the results gathered using the DM ("baseline" result, "channel" result, and "antenna" result) have to be combined: measurement results of the DM and how to combine them [24] are shown graphically in Figure 4. Equation (2) explains itself by graphical summation and subtraction of the results:

$$
\begin{gathered}
10 \log P_{\mathrm{dc}}(y)=10\left[\left(\log P_{\mathrm{ant}}(y)-\log P_{\mathrm{bl}}(y)\right)\right. \\
\left.+\left(\log P_{\mathrm{ch}}(y)-\log P_{\mathrm{bl}}(y)\right)+\log P_{\mathrm{bl}}(y)\right]
\end{gathered}
$$

which leads to

$$
\begin{aligned}
10 \log P_{\mathrm{dc}}(y)= & 10 \log P_{\mathrm{ant}}(y)+10 \log P_{\mathrm{ch}}(y) \\
& -10 \log P_{\mathrm{bl}}(y) .
\end{aligned}
$$

In a linear domain, the result $P_{\mathrm{dc}}(y)$ is computed by

$$
P_{\mathrm{dc}}(y)=P_{\mathrm{ant}}(y) \frac{P_{\mathrm{ch}}(y)}{P_{\mathrm{bl}}(y)} .
$$

The DM result $P_{\mathrm{dc}}(y)$ is ideally the same as the "real world" scenario result $P_{\mathrm{oa}}(y)$ with the deviation $d$ being as small as possible:

$$
10 \log (d)=10 \log \left(P_{\mathrm{dc}}(y)\right)-10 \log \left(P_{\mathrm{oa}}(y)\right) .
$$

2.4. OTA Measurement Time Reduction Using the DM. The "overall" scenario (Figure 1, "real world") with testing fading and radiated performance at once is the ideal setup for measuring the UE, delivering exact results. Using the "overall" setup for a measurement of $N=128$ constellations takes the time $t_{\text {overall }}$. Measuring the UE's behavior on the channel with block fading takes the time $t_{\mathrm{bf} \mathrm{ch}}$, whether radiated for a single constellation or conducted. For the results of the static radiated "antenna" results, it takes the time $t_{\text {ant,st }}$. An example with reasonable time values from practical measurements is given:

$$
t_{\text {overall }}=N \times t_{\text {bf_ch }}=128 \times 20 \mathrm{sec}=2560 \mathrm{sec} .
$$

In comparison, if the measurements are split into conducted and radiated tests, the complete test time needed is $t_{\mathrm{dc}}$ :

$$
\begin{aligned}
t_{\mathrm{dc}} & =1 \times t_{\mathrm{bf}_{-} \mathrm{ch}}+N \times t_{\mathrm{ant}, \mathrm{st}} \\
& =1 \times 20 \mathrm{sec}+128 \times 0.4 \mathrm{sec}=71.2 \mathrm{sec} \\
a & =\frac{2560 \mathrm{sec}}{71.2 \mathrm{sec}} \approx 36 .
\end{aligned}
$$

This is an acceleration of the measurements of a factor $a \approx 36$ ! Therefore measuring the "overall" setup is impractical, and the DM with its splitted measurements shows its strengths. The author uses the "overall" results only for comparison to the DM results.

In the test, data payload is organized in subframes, for example, for the mobile communication standard LTE. The times $t_{\mathrm{dc}}$ and $t_{\text {overall }}$ were measured with a data payload of 400 subframes for the static measurements and 20000 subframes for the faded measurements per measurement or constellation.

If the fading measurements require more subframes, the acceleration rate is even bigger than 36 . This acceleration also reduces the anechoic chamber occupancy. The high side of the DM is that faded measurements have to be done only once, resulting in the mentioned acceleration of test speed.

\section{Signal Model}

The validation of the DM is the goal of this paper. We validate the hypothesis that radiated fading measurements can be split into conducted and radiated measurements and combined afterwards into a single figure of merit. The complete transmission chain is depicted in Figure 6. We can express the received signal vector for a single flat-fading subcarrier as

$$
\mathbf{y}=\mathbf{C}\left(\mathbf{H}_{\mathrm{ant}} \mathbf{H}_{\beta} \mathbf{H}_{\mathrm{ch}} \mathbf{H}_{\alpha} \mathbf{s}+\mathbf{n}\right)
$$

where $\mathbf{s}$ contains the encoded random transmit symbols, $\mathbf{n} \sim \mathbb{C} \mathscr{N}\left\{0, \sigma_{N} \mathbf{I}\right\}$ denotes additive white symmetric complex Gaussian noise, and $\mathbf{H}_{\text {ant }}$ is the antenna matrix, describing the angle dependent complex antenna gain. We assume linear processing employing whether ICD/ZF (inverse channel detector/zero forcing) detector with $\mathbf{C}=\mathbf{H}^{\mathrm{P}}$ or Minimum Mean Square Error (MMSE) detector at the receiver side with $\mathbf{C}=\left(\mathbf{H}^{\mathrm{H}} \mathbf{H}+\sigma_{N}^{2} \mathbf{I}\right)^{-1} \mathbf{H}^{\mathrm{H}}$, where $\mathrm{P}$ is the pseudoinverse and $\mathrm{H}$ is 


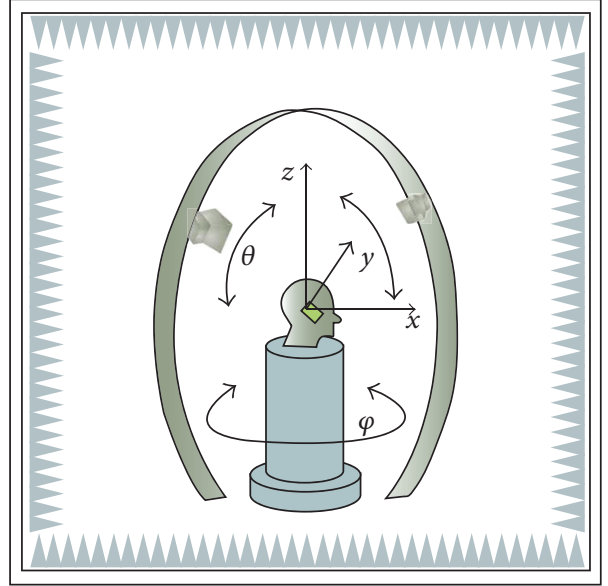

(a) Rotation planes of test antenna and device under test in the anechoic chamber [26]

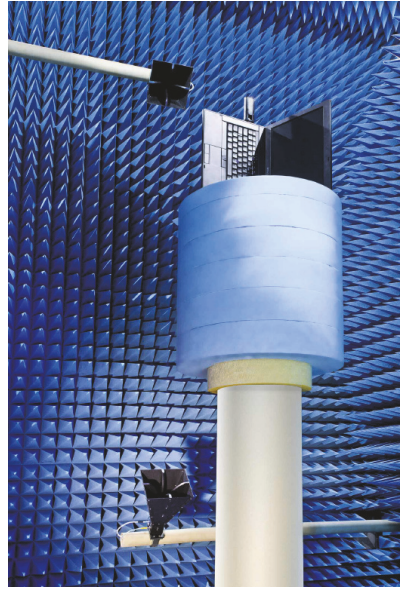

(b) Setup example: test horn antennas and laptop as device under test in the anechoic chamber [26]

FIgURE 5: Test system setup, theoretical and practical.

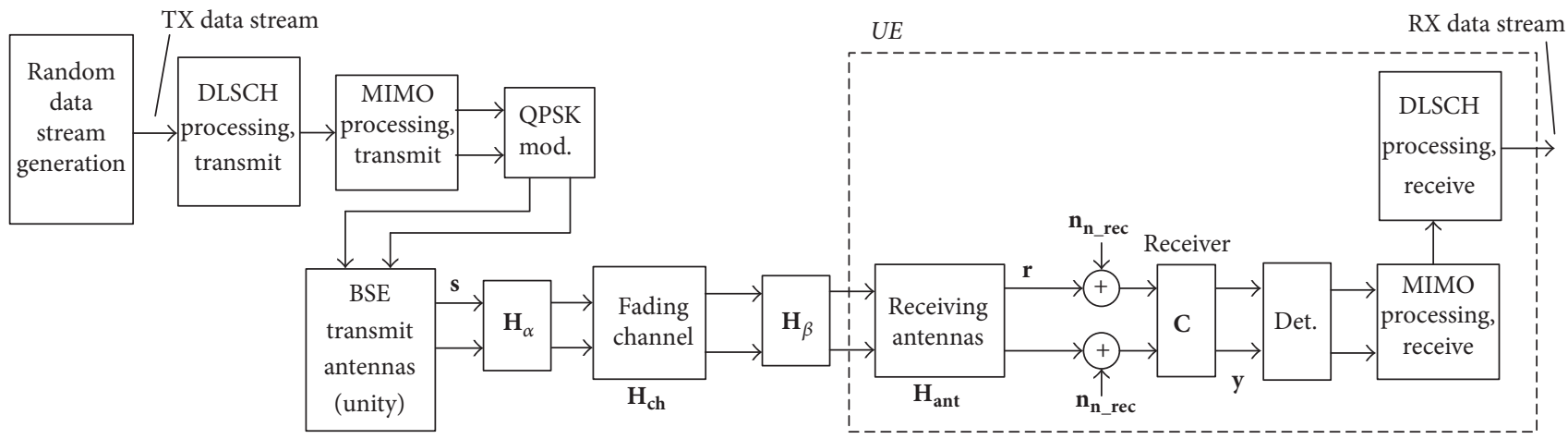

FIGURE 6: Wireless transmission system block diagram for the "overall" simulation including Downlink Shared Channel (DLSCH) processing.

the Hermitian transpose. A general channel matrix is denoted by

$$
\mathbf{H}=\mathbf{H}_{\beta} \mathbf{H}_{\mathrm{ch}} \mathbf{H}_{\alpha}
$$

and $\sigma_{N}^{2}$ is the noise power density. The symbol estimates are demapped and decoded to the RX data stream, as depicted in Figure 6. For uncorrelated channels $\mathbf{H}_{\alpha}=\mathbf{H}_{\beta}=\mathbf{I}$ and $\mathbf{H}=\mathbf{H}_{\mathrm{ch}}$.

3.1. Matrix Models for the Transmission Channels. In general, transmission channels with frequency flat fading have been applied. This results in $2 \times 2$ channel matrices, where each channel element $h_{i j}$ simply represents the complex transmission gain. The authors employ an identity matrix as channel matrix for the "baseline" result

$$
\mathbf{H}_{\mathrm{ch}}=\mathbf{I} \text {. }
$$

All channel power gains are average channel power gains. The channel power gain is represented by the expectation value of the squared Frobenius norm of the channel matrix, which is denoted by $\|\cdot\|_{F}^{2}[31]$

$$
\begin{aligned}
\mathscr{E}\left\{\left\|\mathbf{H}_{\mathrm{ch}}\right\|_{F}^{2}\right\} & =\mathscr{E}\left\{\sum_{i=1}^{2} \sum_{j=1}^{2}\left|h_{\mathrm{ch}, i, j}\right|^{2}\right\} \\
& =\sum_{i=1}^{2} \sum_{j=1}^{2} \mathscr{E}\left\{\left|h_{\mathrm{ch}, i, j}\right|^{2}\right\} .
\end{aligned}
$$

The average power gain of the identity matrix is

$$
\mathscr{E}\left\{\left\|\left[\begin{array}{ll}
1 & 0 \\
0 & 1
\end{array}\right]\right\|_{F}^{2}\right\}=\left\|\left[\begin{array}{ll}
1 & 0 \\
0 & 1
\end{array}\right]\right\|_{F}^{2}=2 .
$$

The average power gain of the Rayleigh fading channel has to be smaller than 2, which reflects the fact that the passive transmission channel has less power gain than the identity channel matrix. The identity channel matrix is physically represented by a wired connection between BSE and UE. 
TABLE 1: Antenna matrices with different coupling and condition number.

\begin{tabular}{lccc}
\hline $\mathbf{H}_{\text {ant }}$ & & $\kappa_{\log } / \mathrm{dB}$ & Coupling between signal paths \\
\hline$\left[\begin{array}{ll}1 & 0 \\
& \end{array}\right]$ & & 0 & No coupling \\
\hline $0.8874\left[\begin{array}{cc}1 & 0.51949 \\
0.51949 & 1\end{array}\right]$ & 10 & Light coupling \\
\hline $0.71414\left[\begin{array}{cc}1 & 0.9802 \\
0.9802 & 1\end{array}\right]$ & 40 & Strong coupling \\
\hline
\end{tabular}

The authors set the average power gain of the Rayleigh fading channel to $v$ :

$$
\mathscr{E}\left\{\left\|\mathbf{H}_{\mathrm{ch}}\right\|_{F}^{2}\right\}=v
$$

where the condition $v<2$ has to be fulfilled. The authors choose $v=0.4096$ arbitrarily, where arbitrary numbers do not necessarily need to be round numbers.

The condition of $v<2$ gives credit to the fact that a transmission over a general channel matrix $\mathbf{H}_{\mathrm{ch}}$ has less energy than a transmission over coaxial cables, represented by $\mathbf{H}_{\mathrm{ch}}=$ I. The second moment for a complex Gaussian distribution $[32]$ is

$$
\Sigma^{2}=\left|h_{\mathrm{ch}, i, j}\right|^{2}=2 \sigma^{2},
$$

where $\sigma$ is the standard deviation of the complex Gaussian distributed channel parameters $h_{\mathrm{ch}, i, j}$ and the expectation value of the squared Frobenius norm of $\mathbf{H}_{\mathrm{ch}}$ is

$$
\begin{aligned}
\mathscr{E}\left\{\left\|\mathbf{H}_{\mathrm{ch}}\right\|_{F}^{2}\right\} & =\sum_{i=1}^{2} \sum_{j=1}^{2} \mathscr{E}\left\{\left|h_{\mathrm{ch}, i, j}\right|^{2}\right\}=\sum_{i=1}^{2} \sum_{j=1}^{2} 2 \sigma^{2} A^{2} \\
& =N_{\mathrm{TX}} N_{\mathrm{RX}} 2 \sigma^{2}=v,
\end{aligned}
$$

where $N_{\mathrm{TX}}$ is the number of transmit antennas and $N_{\mathrm{RX}}$ is the number of receive antennas. With the standard deviation $\sigma=1$, the introduced scaling factor is

$$
A=\sqrt{\frac{\nu}{2 \sigma^{2}}}=\sqrt{\frac{0.4096}{2}}=0.2263 .
$$

All simulations in this paper employ $\mathbf{H}_{\mathrm{ch}}$ including the scaling factor $A=0.2263$ and a complex Gaussian distributed channel with $\sigma=1$.

3.1.1. Kronecker Channel Model. The general correlation matrix of a MIMO channel is defined as

$$
\mathbf{R}=\mathscr{E}\left\{\operatorname{vec}\left(\mathbf{H}_{\mathrm{ch}}\right) \operatorname{vec}\left(\mathbf{H}_{\mathrm{ch}}\right)^{\mathrm{H}}\right\},
$$

where vec $(\mathbf{H})$ stacks the matrix elements in a vector. In this work we use the simplified correlation based Kronecker channel model $[33,34]$

$$
\mathbf{R}=\mathbf{R}_{\alpha} \otimes \mathbf{R}_{\beta},
$$

where the receiver and transmitter correlation matrix [34] are denoted by

$$
\begin{aligned}
& \mathbf{R}_{\alpha}=\left[\begin{array}{ll}
1 & \alpha \\
\alpha & 1
\end{array}\right], \\
& \mathbf{R}_{\beta}=\left[\begin{array}{ll}
1 & \beta \\
\beta & 1
\end{array}\right],
\end{aligned}
$$

respectively. The off-diagonal elements $\alpha$ and $\beta$ define the cross coupling. We use the following parameterization of $\alpha$ and $\beta$ as proposed in $[33,35]$ :

(i) Uncorrelated: $\alpha=0$ and $\beta=0$.

(ii) Medium correlation: $\alpha=0.3$ and $\beta=0.9$.

(iii) High correlation: $\alpha=0.9$ and $\beta=0.9$.

Random correlated channel realizations are computed according to

$$
\mathbf{H}_{\mathrm{ch}}=\mathbf{R}_{\beta}^{1 / 2} \mathbf{H}_{\mathrm{w}} \mathbf{R}_{\alpha}^{1 / 2},
$$

where $\mathbf{H}_{\mathrm{w}}$ denotes a MIMO matrix with independent and identically distributed (i.i.d.) complex Gaussian entries $\mathbb{C} \mathcal{N}\{0,1\}$ with zero mean and variance one.

3.1.2. Antenna Matrices. We assume no coupling between the base-station antennas. The DUT antenna coupling is denoted by $\mathbf{H}_{\text {ant }}$. It is calculated using the complex E-field pattern [26]:

$$
\mathbf{H}_{\text {ant }}\left(\Omega_{1}, \Omega_{2}, p, q\right)=\left[\begin{array}{ll}
E_{p 1}\left(\Omega_{1}\right) & E_{q 1}\left(\Omega_{2}\right) \\
E_{p 2}\left(\Omega_{1}\right) & E_{q 2}\left(\Omega_{2}\right)
\end{array}\right],
$$

$$
\{p, q \in \Theta, \Phi\}
$$

where $E$ denotes the complex $E$-field pattern of the receiving antenna 1 or 2 . We denote the polarization instances of the test antennas with $\{p, q\}$. These polarization instances $\{p, q\}$ can be in $\Phi$ - or in the $\Theta$-plane.

Matrix $\mathbf{H}_{\text {ant }}$ is shown in Table 1 for three exemplary antennas with different condition numbers $\kappa_{\text {log }}$, namely, for fully decoupled antennas $(\kappa=0 \mathrm{~dB})$ to strongly coupled antennas $(\kappa=40 \mathrm{~dB})$. The antenna matrix $\mathbf{H}_{\text {ant }}$ is normalized to a squared Frobenius Norm (SQFN) of 2:

$$
\left\|\mathbf{H}_{\mathrm{ant}}\right\|_{F}^{2}=\sum_{i=1}^{2} \sum_{j=1}^{2}\left|h_{\mathrm{ant}, i j}\right|^{2}=2,
$$




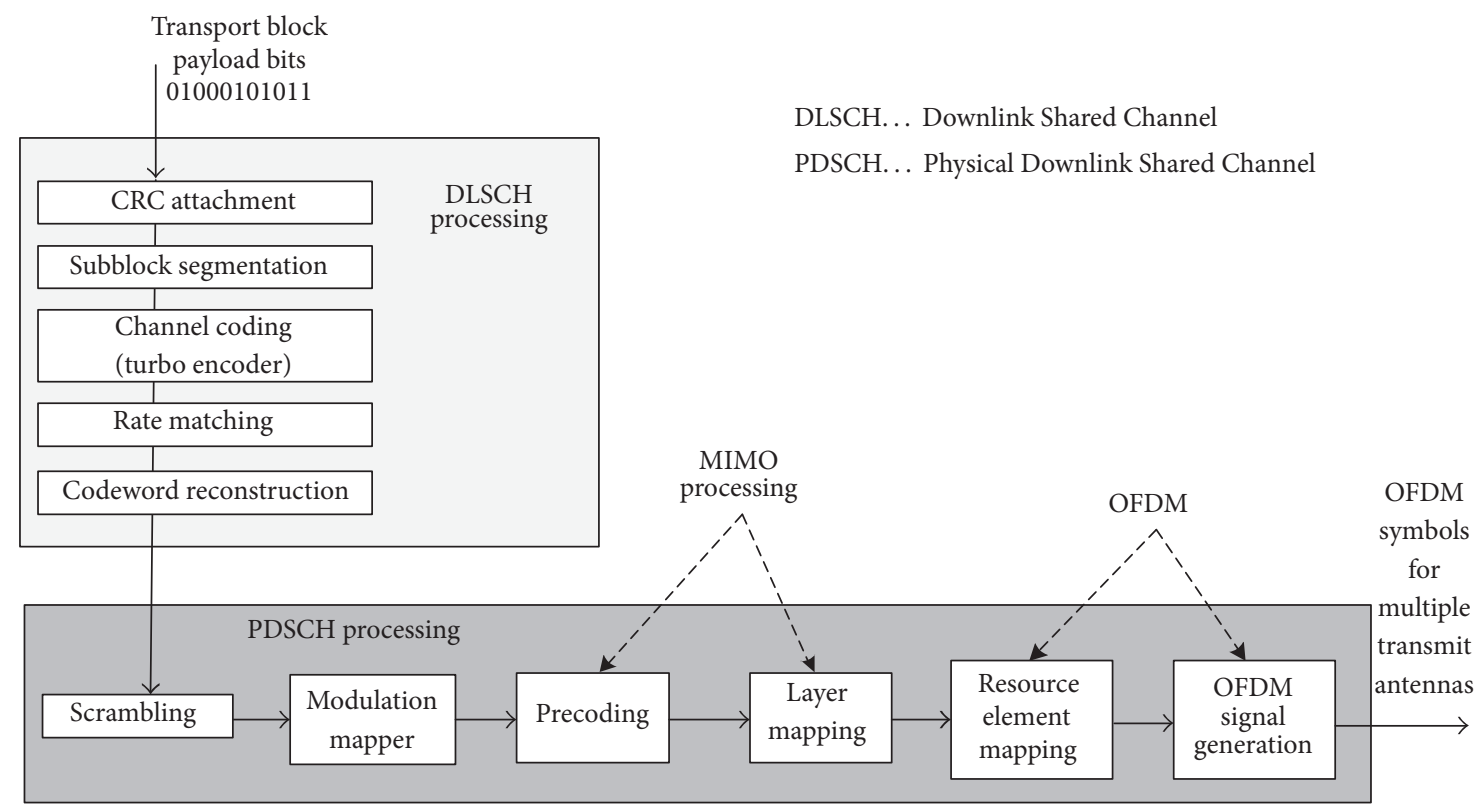

FIGURE 7: LTE signal processing block diagram for transmit diversity and spatial multiplexing (MIMO) [27] in the transmitter.

which corresponds to the power gain of an identity matrix, shown in (11). The reason is that results can be compared easily, if the power gain is the same for all $\mathbf{H}_{\text {ant }}$. The antenna condition number in linear terms

$$
\kappa_{\text {lin }}=\frac{\sigma_{\max }}{\sigma_{\min }}
$$

is the ratio of the largest to the smallest eigenvalue of the antenna matrix $\mathbf{H}_{\text {ant }}\left(\Omega_{1}, \Omega_{2}, p, q\right)(23)$.

The bigger $\kappa_{\text {lin }}$ is, the worse the matrix conditioning is. For visualization purposes the authors define

$$
\kappa_{\log }=20 \log _{10}\left(\kappa_{\operatorname{lin}}\right) \text {. }
$$

The identity antenna matrix corresponds to fully decoupled antennas, with $\kappa_{\log }=0 \mathrm{~dB}$. Increasing coupling between the antennas provide antenna matrices with higher condition numbers.

\subsection{LTE as a Showcase for MIMO Enabled Communication} Standards. The wireless mobile communication standard Long Term Evolution (LTE) is a common use case for the application of the DM. Therefore main elements of the standard are used for the numerical validation.

3.2.1. Modeling of the Physical (PHY) Layer for Downlink/Transmitter Side. Figure 7 shows a block diagram of LTE transmitter [27]. User payload is segmented into blocks; then channel coding with rate matching is applied. LTE employs turbo coding for channel coding, and code blocks are concatenated to create codewords. The user data is scrambled, which is an operation where the user data is convolved with a pseudo random sequence resulting in the scrambled bit stream. In the modulation mapper the bit stream is mapped to a certain complex in phase/quadrature phase (I/Q) modulation scheme, like (i) QPSK (Quadrature Phase Shift Keying), mapping 2 bits to one symbol

(ii) 16 QAM (16-Quadrature Amplitude Modulation), mapping 4 bits to one symbol

(iii) 64 QAM (64-Quadrature Amplitude Modulation), mapping 8 bits to one symbol

In the MIMO processing the complex modulated data are shaped for the different MIMO subchannels according to the available antennas and the transmit mode. The Orthogonal Frequency Division Multiplexing (OFDM) processing takes care of the data placement in the domains frequency and time (Resource Element (RE) Mapping and OFDM signal generation). The data are amplified and transmitted by the multiple antennas.

3.2.2. Channel Estimation Errors. Another part of the investigations is to have a closer look on channel estimation errors. In consideration of receiver types like the ICD/ZF or the MMSE, it is assumed that the transmission channel is perfectly known. The following investigations also consider imperfect knowledge of the channel taking into account the length of the pilot signals that are used for channel estimation. Mengali proposed a method to compute the variance of the estimation error for QPSK. The error follows a normal distribution with a first moment equaling zero and variance [36]

$$
\sigma_{\text {est_err }}^{2}=\frac{1}{L} \frac{1}{P / N_{0}} \text {, }
$$

where $L$ is the length of the pilot sequence and $P /\left(B N_{0}\right)$ is the symbol energy to the noise energy (SNR). The channel estimation in LTE is executed over 19 OFDM subcarriers and 
10 subframes, each containing 2 slots. Every slot contains one symbol for estimation. This results in

$$
\begin{aligned}
L= & 19 \text { subcarriers } \times 10 \text { subframes } \times 2 \text { slots } \\
& \times 1 \text { symbol }=380 \text { symbols }
\end{aligned}
$$

per estimation [3, 35]. With (25) and (26), the channel estimation error variance $\sigma_{\text {est_err }}^{2}$ can be calculated as

$$
\sigma_{\text {est_err }}^{2}=\frac{1}{380} \frac{1}{P / N_{0}} \text {. }
$$

Equation (25) shows the dependency of the estimation error on the symbol energy to noise energy $P / N_{0}$. The equation is valid for flat-fading and time invariant channels.

\section{DM Validation: MIMO Channel Capacity Approach}

4.1. Validation Procedure. As an additional step, the authors employ the MIMO channel capacity expression; therefore a generic view can be achieved in the investigations. This approach imposes the use of a receiver of general type working close to or at the Shannon bound. For the investigations a $2 \times 2$ MIMO transmission system with two uncorrelated data streams and symbol energy of $P / N_{\mathrm{T}}$ per stream is chosen. The symbol energy is equally distributed over the two transmission paths. The signal covariance matrix of the symbols

$$
\mathbf{R}_{\mathrm{ss}}=\frac{P}{N_{\mathrm{T}}} \mathbf{I}_{N_{\mathrm{T}}}
$$

is a diagonal matrix in this case [37], where $N_{\mathrm{T}}$ is the number of transmit antennas. The sum of the main diagonal elements of $\mathbf{R}_{\mathrm{ss}}$ is the symbol energy [37]:

$$
\operatorname{trace}\left(\mathbf{R}_{\mathrm{ss}}\right)=P \text {. }
$$

Using these assumptions, the rate of the MIMO channel simplifies to [37]

$$
C=\mathscr{E}\left\{\log _{2} \operatorname{det}\left(\mathbf{I}_{N_{\mathrm{R}}}+\frac{P}{N_{\mathrm{T}} N_{0}} \mathbf{H H}^{\mathrm{H}}\right)\right\},
$$

where $N_{\mathrm{R}}$ is the number of receiving antennas, $\mathbf{H}$ is the channel matrix, the trace operator denotes the sum of the main diagonal elements of a matrix, $\mathbf{I}_{N_{\mathrm{R}}}$ is the identity matrix with dimension $N_{\mathrm{R}} \times N_{\mathrm{R}}, \mathscr{E}$ is the expected value, and det is the determinant, respectively. For the sake of simplicity, the validations in this section use the absolute throughput rate $C$ and not the relative throughput rate $y$ as in Section 2.3. In both cases the validity of the Decomposition Method can be shown.

Equation (30) can also be expressed in terms of the eigenvalues of $\mathbf{H H}^{\mathrm{H}}$ employing the eigendecomposition [38]

$$
C=\mathscr{E}\left\{\log _{2} \operatorname{det}\left(\mathbf{I}_{N_{\mathrm{R}}}+\frac{P}{N_{\mathrm{T}} N_{0}} \mathbf{Q} \boldsymbol{\Lambda} \mathbf{Q}^{\mathrm{H}}\right)\right\}
$$

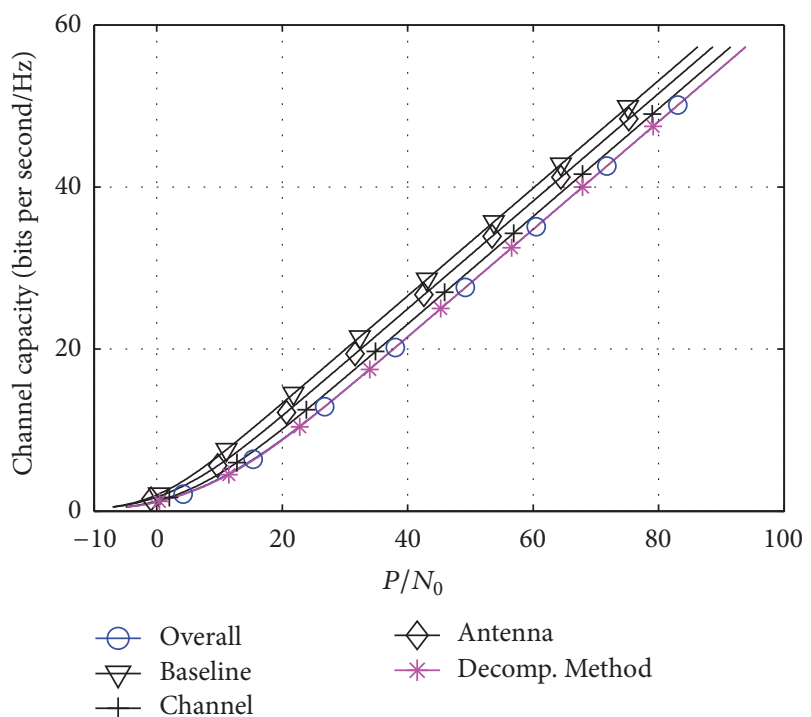

FIgURE 8: Absolute channel capacity per bandwidth versus $P / N_{0}$ using DM; $\kappa_{\mathrm{H}_{\text {ant }}}=10 \mathrm{~dB}$. All measurements including the ideal and the real result are exhibited. "Overall" and DM results are almost overlapping.

reducing to

$$
C=\mathscr{E}\left\{\log _{2} \operatorname{det}\left(\mathbf{I}_{N_{\mathrm{R}}}+\frac{P}{N_{\mathrm{T}} N_{0}} \boldsymbol{\Lambda}\right)\right\},
$$

where $\Lambda$ contains the nonnegative eigenvalues on its main diagonal $[37,39]$.

Using the eigenvalue matrix $\Lambda$ and its elements $\lambda_{i}$, the expected (ergodic) rate can also be written as [39]

$$
C=\mathscr{E}\left\{\sum_{i=1}^{N_{\mathrm{R}}} \log _{2}\left(1+\frac{P}{N_{\mathrm{T}} N_{0}} \lambda_{i}\right)\right\} .
$$

We use (30) to calculate the ergodic rate for the three DM scenarios and the overall scenario numerically. The channel matrix is modified according to the different steps of the DM as follows:

(i) “Overall” transmission scenario (oa): $\mathbf{H}=\mathbf{H}_{\mathrm{ant}} \mathbf{H}_{\mathrm{ch}}$.

(ii) "Antenna" transmission scenario (ant): $\mathbf{H}=\mathbf{H}_{\text {ant }}$.

(iii) "Channel" transmission scenario (ch): $\mathbf{H}=\mathbf{H}_{\mathrm{ch}}$.

(iv) "Baseline" transmission scenario (bl): $\mathbf{H}=\mathbf{I}$.

The required transmit energy $P$ is a function of the channel rate $C$ (for fixed noise power spectral density $N_{0}$ ) for a given set of channel matrices, providing $P_{\mathrm{oa}}(C), P_{\text {ant }}(C), P_{\mathrm{ch}}(C)$, and $P_{\mathrm{bl}}(C)$. Inserting these results in (2) and (4) we can compute the deviation $d$.

Figure 8 exhibits the absolute throughput rate $C$ versus $P / N_{0}$ for different antenna correlation condition numbers $(\kappa=10 \mathrm{~dB})$. In theory, the channel rate $C$ does not have a limit, as long as $P / N_{0}$ is rising. In practical systems the throughput will always saturate at the maximum data rate related to the chosen modulation and coding scheme of a 


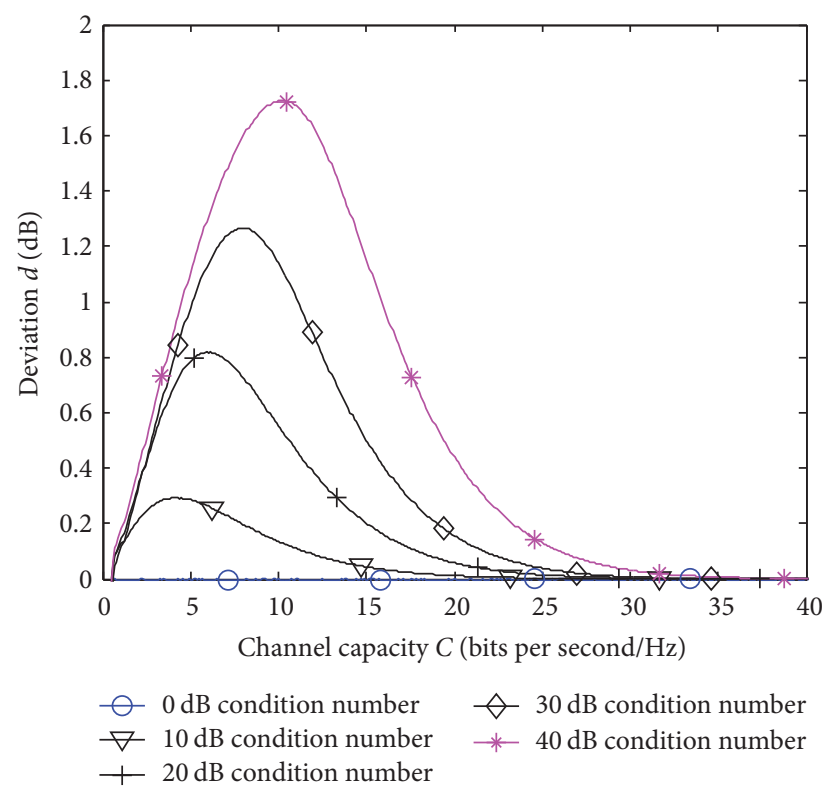

FIGURE 9: Deviation $d$ versus absolute channel capacity $C$ using DM for $\kappa_{\mathrm{H}_{\mathrm{ant}}}=\{0 \mathrm{~dB}, 10 \mathrm{~dB}, 20 \mathrm{~dB}, 30 \mathrm{~dB}, 40 \mathrm{~dB}\}$.

wireless transmission. Figure 8 also shows that the DM result does not deviate at $\kappa=0 \mathrm{~dB}$. The deviation $d$, as defined in (4), is increasing with rising $\kappa$ and is also depending on $P / N_{0}$.

The deviation $d$ versus the channel capacity $C$ is depicted in Figure 9. The plots show $d$ over $C$ for $\kappa \in$ $\{0 \mathrm{~dB}, 10 \mathrm{~dB}, 20 \mathrm{~dB}, 30 \mathrm{~dB}, 40 \mathrm{~dB}\}$. For $\kappa_{\mathrm{log}}=0 \mathrm{~dB}$, the deviation $d$ is zero, and the DM works perfectly. For rising $\kappa, d$ is increasing and the maximum of $d$ shifts towards bigger $P / N_{0}$ and decreases slower than at lower values of $\kappa$. Nevertheless, even at a very bad conditioned antenna matrix with $=40 \mathrm{~dB}$, the maximum deviation $d$ is below $1.72 \mathrm{~dB}$.

4.2. Conclusion on the Channel Capacity Approach. The authors validate the DM by using the MIMO channel capacity formula. MIMO channel capacity curves are simulated and overlaid using the DM. This section compares the "overall" results to the "DM" results by showing the occurring deviations $d$. For the given antenna condition numbers the DM works within the error margin given in Figure 9. This error margin of $d$ lies within the error margin given by measurement uncertainties in practical OTA measurements [40]. Dozens of measurement uncertainty sources are listed in [41, 42]: mismatch of transmitter chain, insertion loss of transmitter chain, influence of the probe antenna, uncertainty of BSE power level, statistical uncertainty of throughput measurement, fading flatness within the LTE band, uncertainty of the network analyzer, and instability of cable connections just to name a few.

\section{DM Validation: Numerical Analysis Approach}

5.1. Introduction. To validate the DM from another perspective, the authors choose the numerical link level simulation of a wireless MIMO transmission system. The DM is intended for testing general UE employing MIMO antenna technology. An important point is to investigate the validity of the DM for the use with LTE devices. It will be a main application of the method. As the number of employed signal processing elements for LTE is high, this paper focuses on the most important blocks of the transmission in the PHY layer for the simulation and numerical validation.

The authors again realize a transmission using the block diagrams in Figure 6 for the numerical simulation of the transmission. They discuss the results of this transmission system and present the validation of the DM under the given conditions.

5.2. Concept of the Numerical Simulations. As LTE is a complex transmission protocol containing a lot of different functionality blocks, it is necessary to focus on the blocks that might be influential on the DM. All investigations are done with flat-fading wireless transmission channels. If problems show up already with the simple model of flat-fading channels, all scenarios containing more complex transmission models including tapped delay channel models and OFDM schemes will also have a problem.

A wireless system, employing an OFDM scheme, splits the complete channel bandwidth of the frequency dependent channel in many narrow-band channels that are assumed to be of flat-fading nature. In the following investigations, the authors deal with one of these small flat-fading OFDM channels. This has the consequence that it is not necessary to simulate all the OFDM channels. All steps in the DLSCH block (Figure 7) are implemented, as well as the modulation mapper and the MIMO processing blocks. The elements in the Physical Downlink Shared Channel (PDSCH) like scrambling, RE mapping, and OFDM processing have been omitted, as the authors expect minor influence on the results when employing frequency flat transmission channels.

5.3. Simulation Setup Configuration and Figures of Merit. The parameters for the simulation setup and the necessary figures of merit (FOMs) are introduced in this section, as well as how they can be calculated. The block diagram of the transmission systems in Figure 6 eases the understanding of the parameters and the FOMs.

5.3.1. Transmission Data Block Size and Subframe Error Rate (SFER). The transmitted data streams have a length of $2^{8}$ bits per realization of the channel matrix $\mathbf{H}_{\text {ant }}$. The transmitted (TX) bit stream is compared to the received bit stream (RX). This is done with a test window size of $2^{4}$ bits, so-called subframes (SFs). If the RX subframe completely matches the TX subframe, it is recognized as correct. If there are one or more bits wrong, the complete subframe is rated to be incorrect. The mathematical formulation of the Subframe Error Rate (SFER) is

$$
\text { SFER }=\frac{\text { number of correctly received SFs }}{\text { number of all transmitted SFs }},
$$

and it ranges from 1 (no subframe (SF) transmitted correctly) to 0 (all SFs transmitted correctly). 
5.3.2. Relative Throughput (RTP). The relative throughput is

$$
\text { RTP }=1-\text { SFER } .
$$

It ranges from 0 (no SF transferred correctly) to 1 (all SFs transferred correctly).

Downlink Power, Bandwidth, and Noise. The downlink power used for the simulation ranges from $-130 \mathrm{dBm}$ to $-50 \mathrm{dBm}$. It is always related to a subcarrier bandwidth of $B=15 \mathrm{kHz}$. The noise energy is

$$
\begin{aligned}
N_{0} & =-174 \mathrm{dBm}+10 \log _{10}(B)+N_{f} \\
& =-174 \mathrm{dBm}+10 \cdot \log _{10}(15 \mathrm{kHz})+5.2 \mathrm{~dB} \\
& =-127 \mathrm{dBm},
\end{aligned}
$$

where $N_{f}=5.2 \mathrm{~dB}$ is the noise figure of the UE employed for the measurements in this paper; white noise is assumed.

5.3.3. Simulation Parameters and Number of Realizations. The numerical simulations employ a variation of settings:

(i) Detector type: ICD/ZF or MMSE

(ii) Ideal or nonideal channel estimation

(iii) DLSCH processing enabled/disabled

(iv) Low, medium, or high correlation matrices $\mathbf{H}_{\alpha}$ and $\mathbf{H}_{\beta}$

(v) Receive-antenna condition number $\kappa$

The authors implemented the transmission chain following the block diagram in Figure 6 for the simulations with DLSCH processing without DLSCH processing.

A realization is a certain transmission system instance employing one set of stochastic parameters, like channel parameters or channel estimation errors. The authors executed all simulations with 5000 realizations of the channel matrix $\mathbf{H}_{\mathrm{ch}}$. They averaged the throughput of all realizations linearly for each downlink power value throughout this paper. This averaged throughput is displayed in the result throughput versus downlink power curves. Every realization employs a new set of transmission data blocks.

\subsection{Results}

5.4.1. Relative Throughput versus Downlink Power Curves. Figure 10 exhibits an example for a set of simulation result curves with relative throughput over downlink power. The mentioned result figure employs an antenna condition number of $\kappa=40 \mathrm{~dB}$ and other different transmission parameters, as mentioned in the caption of the figures.

The exemplarily taken graph shows all three necessary curves to calculate the DM result: the "baseline"; "channel"; and "antenna" curves. The DM result and the "overall" result are also depicted. If the DM works well within the given parameters, the DM result and the "overall" result overlap, as it is the case in Figure 10. The quantitative measure of how well the DM works is the deviation $d$. It shows the

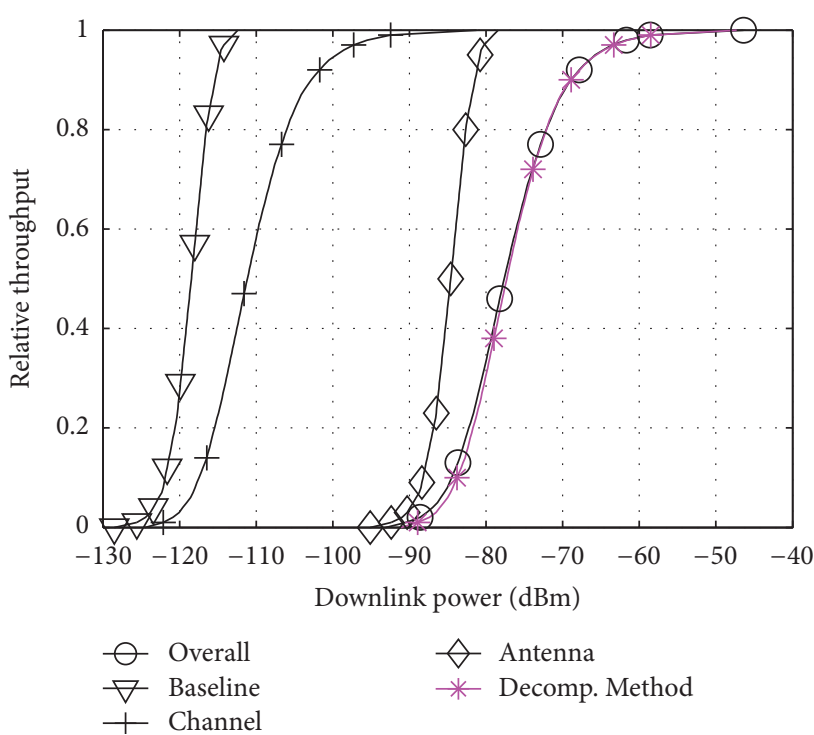

FIGURE 10: Simulation result example: downlink power versus relative throughput; ICD/ZF receiver, DLSCH processing on, ideal channel estimation, correlation low, and antenna condition number $40 \mathrm{~dB}$.

difference between the actual value (DM result) and the ideal value ("overall" result), as depicted in (4). The authors simulated the different transmission scenarios for a wide range of parameters.

All downlink power values versus RTP show the same "baseline" result curve, which exhibits the best performance a transmission system can reach. The higher the performance is, the more a certain throughput can be received with less downlink power. The BSE is directly connected to the receiver: $\mathbf{H}_{\beta} \mathbf{H}_{\mathrm{ch}} \mathbf{H}_{\alpha}=\mathbf{I}$.

The slope of the "channel" curve is less steep in comparison to the "baseline" curve, because of the complex normal distribution of the channel parameters in a general $\mathbf{H}_{\mathrm{ch}}$ matrix.

In the case that the receiving antenna matrix is an identity matrix and uncorrelated $(\kappa=0 \mathrm{~dB})$, the "antenna" result overlaps with the "baseline" result. The "antenna" simulations employ $\mathbf{H}_{\beta} \mathbf{H}_{\mathrm{ch}} \mathbf{H}_{\alpha}=\mathbf{I}$. The overlapping of the "overall" curve and the DM curve is the ideal case, and in this case the DM works perfectly.

As the antenna elements start to be correlated (condition number $\kappa$ of the antenna matrix $\mathbf{H}_{\text {ant }}>0 \mathrm{~dB}$ ), the receiver performance is degraded. This degradation leads to a result curve (the "antenna" result curve) that is a right shifted version of the "baseline" result curve, as shown in Figure 10. This right-shift means that the receiver needs more signal downlink power to reach the same throughput as with an uncorrelated channel. The reason for this behavior is that the receiver cancels the influence of the channel, but it also enhances the existing intrinsic noise, generated by the RF front-end of the UE.

The more the receiving antennas are coupled, the worse the conditioning of the antenna matrix is (indicated by $\kappa$ ). Looking at Figure 10, the antenna matrix condition number 


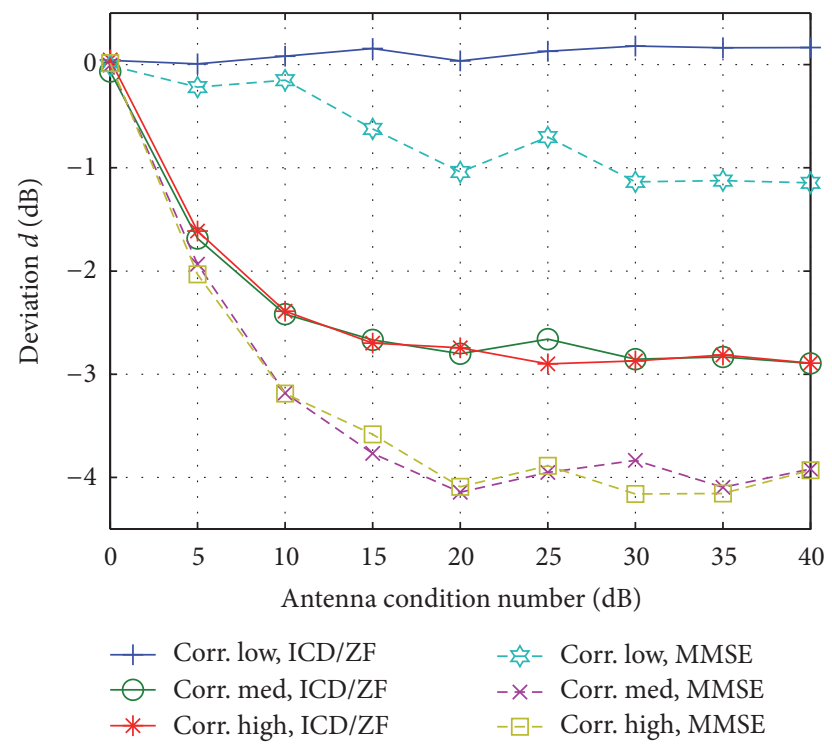

FIGURE 11: Deviation $d$ versus antenna condition number for DLSCH processing on, nonideal channel estimation; low, medium, and high channel correlation; ICD/ZF and MMSE receiver.

$=40 \mathrm{~dB}$. For a relative throughput of 0.7 , the "baseline" setup needs a downlink power of $-117 \mathrm{dBm}$, while the "antenna" setup needs $-83 \mathrm{dBm}$, which is a difference of $34 \mathrm{~dB}$.

So to say, the degradation through the correlation of the receiving antennas results in a degraded performance of the whole transmission system. The influence of the different simulation parameters will be discussed in the following section.

5.4.2. Influence of the Transmission System Parameters on the Deviation $d$. The authors show the influence of the simulation parameters on the deviation $d$. These results are exhibited in Figures 11-16 and the authors will discuss them elaborately. The different simulation parameters are

\section{(i) DLSCH processing on/off}

(ii) ideal/nonideal channel estimation

(iii) low, medium, and highly correlated channel parameters

These figures show the investigation of the deviation $d$ versus the antenna condition number $\kappa$ for this set of parameters. They are arranged in different ways to highlight certain ways of behavior. Certain curves are exemplarily taken to explain a certain way of behavior. Some facts are mentioned twice, to ease understandability. Please note that the depicted deviations $d$ are maximum values that will not be reached in practice, when a real antenna system is employed.

Influence of the Condition Number $\kappa$. Generally speaking, the absolute deviation $|d|$ rises with rising antenna condition number $\kappa$. Figure 11 is taken exemplarily to show this behavior, which appears in all simulations. The absolute deviation starts to grow rapidly and settles more or less at $=$ $20 \mathrm{~dB}$. The employed simulation parameters for the deviation

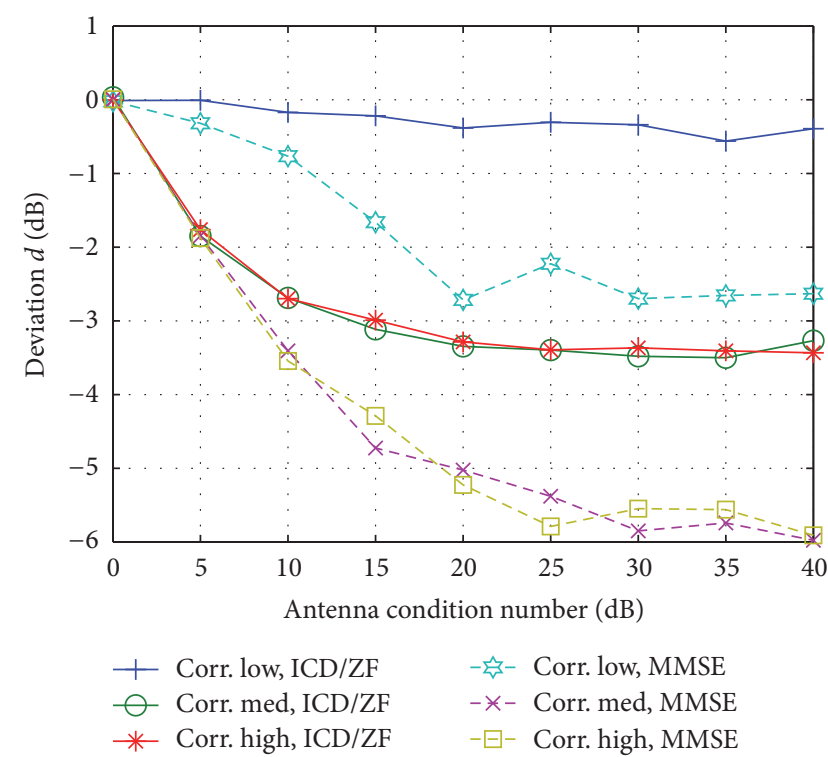

FIGURE 12: Deviation $d$ versus antenna condition number for DLSCH processing off, nonideal channel estimation; low, medium, and high channel correlation; ICD/ZF and MMSE receiver.

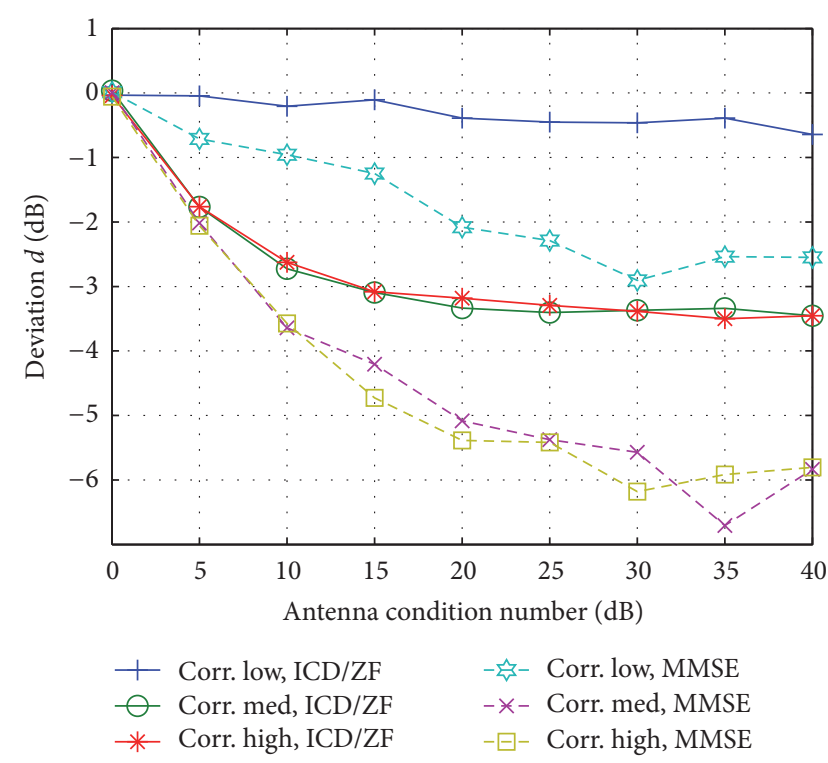

FIGURE 13: Deviation $d$ versus antenna condition number for DLSCH processing off, ideal channel estimation; low, medium, and high channel correlation; ICD/ZF and MMSE receiver.

results in Figure 11 are as follows: DLSCH processing on, nonideal channel estimation; low, medium, and high channel correlation; ICD/ZF and MMSE receiver.

It is interesting to see that the DM works almost perfectly for low correlation and the ICD/ZF receiver. In comparison, the MMSE receiver causes an approximate maximum deviation $d=-1 \mathrm{~dB}$ at low correlation. With rising correlation, both receiver types, MMSE and ICD/ZF, cause bigger absolute deviation $|d|$. The value of $|d|$ is greater for MMSE than for ICD/ZF in general. 


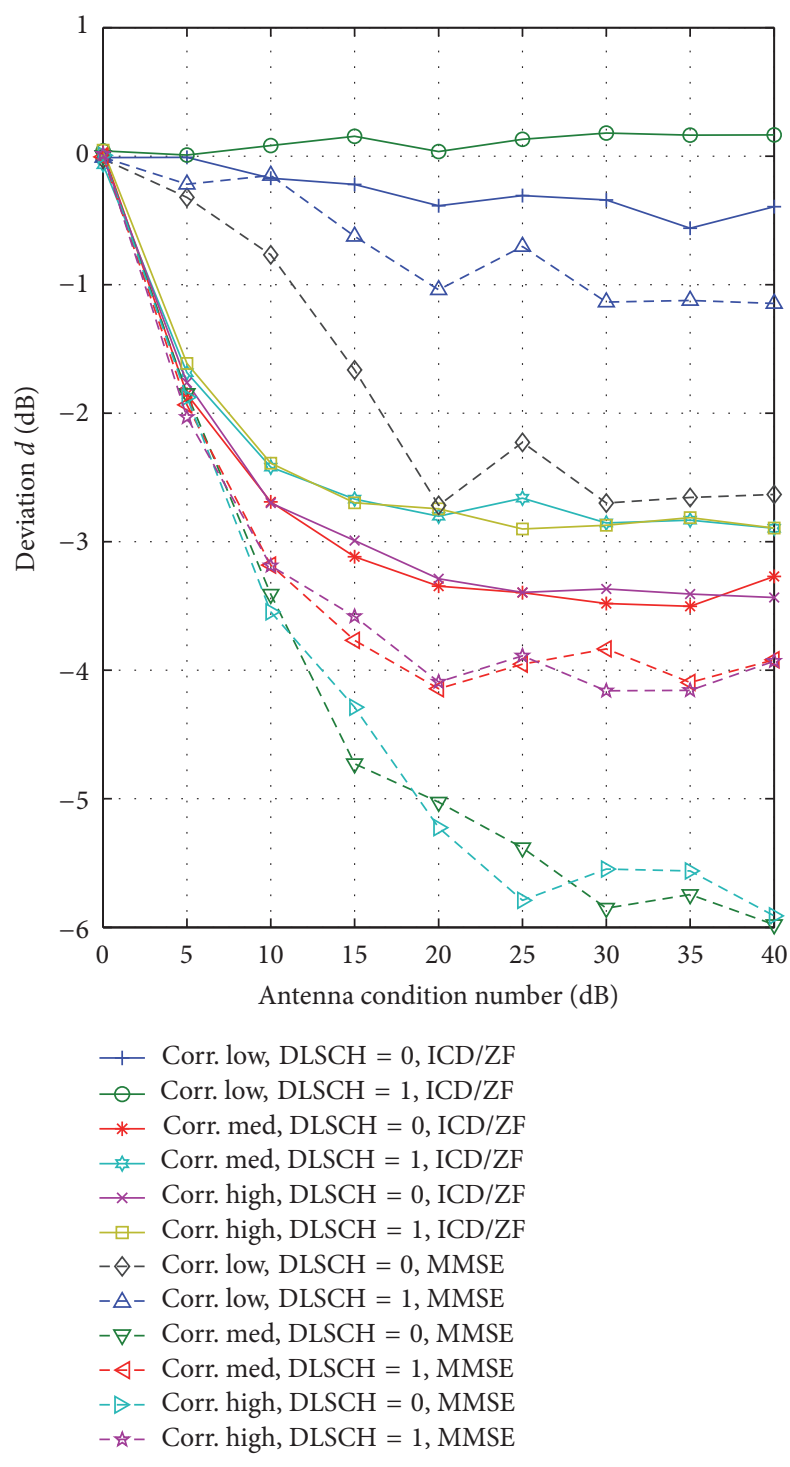

FIgURE 14: Deviation $d$ versus antenna condition number for DLSCH processing on/off, nonideal channel estimation; low, medium, and high channel correlation; ICD/ZF and MMSE receiver.

Influence of the Channel Correlation. Figure 11 also points out the differences of the different channel correlations low, medium, and high. DLSCH processing is applied and the channel estimation is nonideal. The DM works almost perfectly for ICD/ZF receivers at low correlations.

In contrast, the receiver type MMSE delivers mentionable deviations already at low channel correlations. As soon as channel correlation becomes medium or high, the absolute deviation $|d|$ increases. The deviation $|d|$ is always bigger for the MMSE receiver than for the ICD/ZF receiver. Figure 12 shows the same trends but with higher absolute deviation $|d|$ (no DLSCH processing is applied).

Influence of DLSCH Processing on/DLSCH Processing off. Figure 16 depicts the differences of the deviation $d$, between

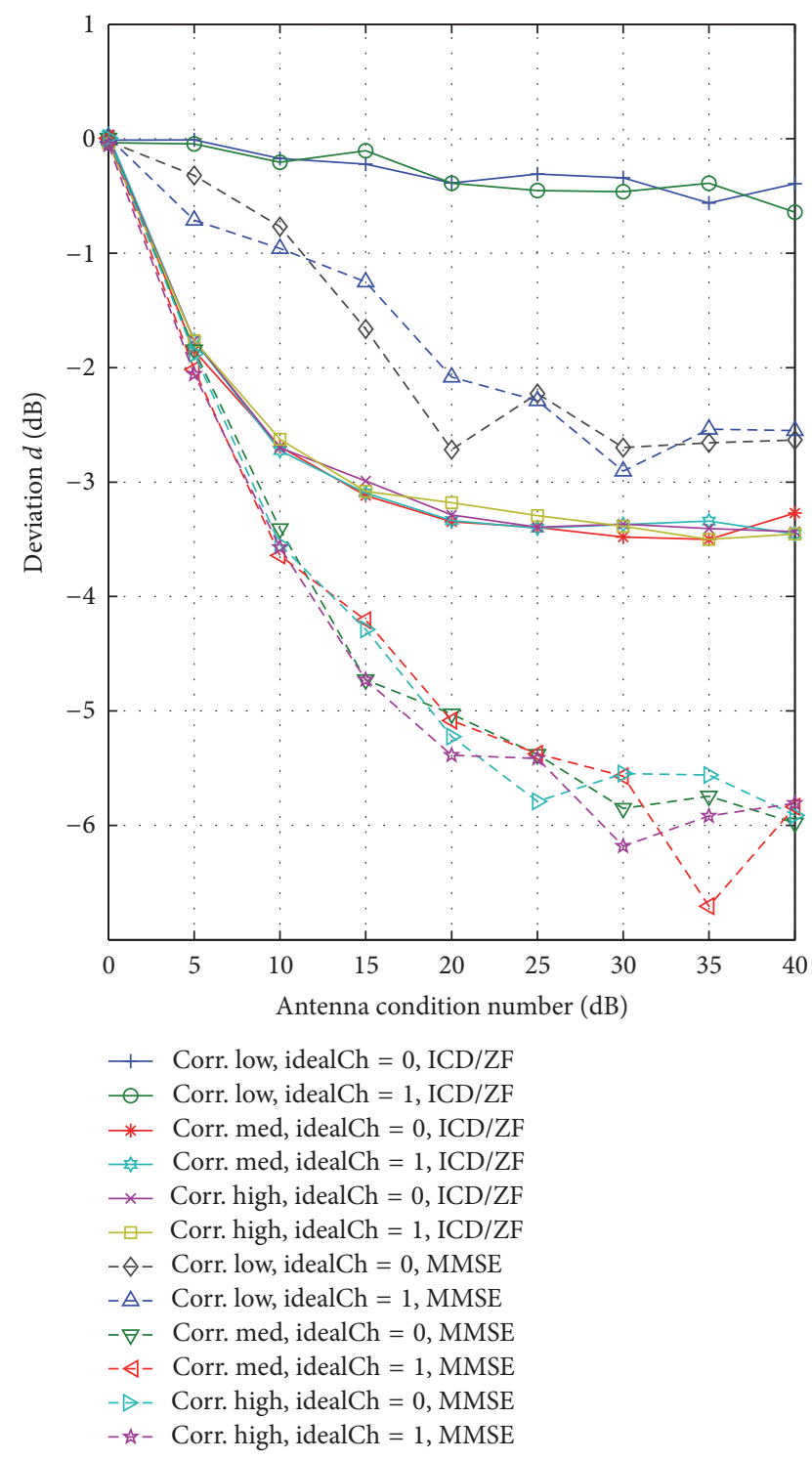

FIgure 15: Deviation $d$ versus antenna condition number for DLSCH processing off, nonideal/ideal channel estimation; low, medium, and high channel correlation; ICD/ZF and MMSE receiver.

DLSCH processing being turned on and off for ideal channel estimation. The channel correlations are again low, medium, and high.

In contrast, Figure 14 exhibits the results for DLSCH processing being turned on and off for nonideal channel estimations. The main message of both graphs is that if DLSCH processing is turned on, the abs. deviation $|d|$ becomes smaller for all three correlation types and two receiver types (MMSE and ICD/ZF), in comparison to DLSCH processing turned off.

This is true for all channel correlation types: low, medium, and high. This is the reason why the DM is very suitable for LTE performance tests, as LTE always uses DLSCH processing. The influence of the channel estimation on the results of $d$ is very small. 


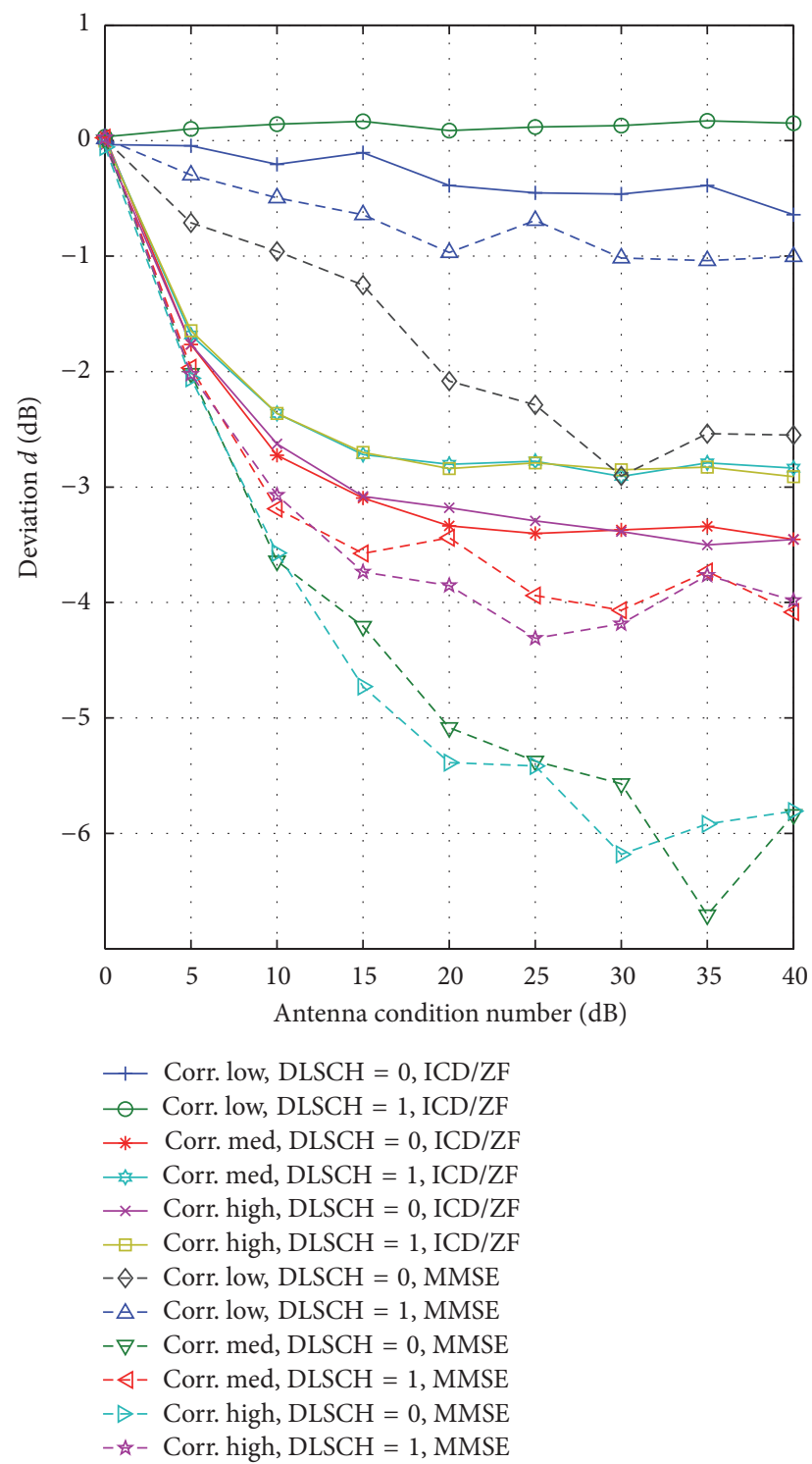

FIGURE 16: Deviation $d$ versus antenna condition number for DLSCH processing on/off, ideal channel estimation; low, medium, and high channel correlation; ICD/ZF and MMSE receiver.

Summarizing, the following facts can be pointed out:

(i) The DM suits well its purpose, the performance testing of MIMO enabled wireless UE, especially for low correlation channels, and it delivers small deviations $d$ for both receiver types: ICD/ZF and MMSE.

(ii) In general, if the antenna condition number $\kappa$ is rising, the absolute deviation $|d|$ rises.

(iii) If channels correlations are medium or high, the absolute deviation $|d|$ is bigger than that for low correlation channels.

(iv) The DM delivers better results with the ICD/ZF receiver in comparison to the MMSE receiver for all channel correlations. (v) The influence of the channel estimation error is negligible in the case of LTE pilot sequence length as a reference example.

(vi) If a transmission system employs DLSCH processing, the DM performs better compared to being without it. This behavior makes it very suitable for performance tests with LTE, as it always uses DLSCH processing.

(vii) Rising deviation occurs for antennas with very poor behavior, but manufacturers will strongly avoid such poor designs as it results in a significantly reduced MIMO system performance.

All diagrams with deviation $d$ versus antenna condition number $\kappa$ show maximum errors, which will not occur in practice. The reason is that the condition number of the antenna matrix $\mathbf{H}_{\text {ant }}$ is different for every constellation. Therefore the authors execute investigations with a practical antenna set and the mean deviation $\bar{d}$ in the following chapter.

5.4.3. Investigating the Deviation $d$ Employing LTE Reference Antennas. The deviation $d$ versus condition number $\kappa$ graphs tend to overestimate practically arising deviations, as UE manufacturers do not intentionally build very poor performing antenna systems. For the reason of better visualization the authors investigated the distribution of the deviation $d$ with LTE reference antennas with designated performances.

The company Motorola Mobility designed LTE reference antennas for the purpose of interlaboratory measurement campaigns executed in the COST 1004 actions [28]. The printed circuit board contains the antennas and a metal housing. Different UE can be placed inside the metal cavity. The UE can be connected to the external reference antenna elements with coaxial cables, and the internal antennas are deactivated. A picture of the reference antenna is exhibited in Figure 17.

It is available in three different performance types:

(i) "Good" performance

(ii) "Nominal" performance

(iii) "Bad" performance

The performance naming refers to the coupling of the antenna elements in the different reference antenna versions. This coupling is expressed with condition number $\kappa$, which is different for every constellation. A constellation contains test antenna positions and polarization, as well as UE placement.

The authors use the 128 constellations [30] for statistical investigations on the logarithmic antenna condition numbers $\kappa$. Every single constellation delivers a certain $\kappa$, which can be calculated employing the E-field antenna pattern as shown in Section 3.1.2. Figure 18 shows the distribution of $\kappa$ for the 128 constellations for all three antenna types. Also the mean logarithmic value of the condition number

$$
\bar{\kappa}=\frac{\sum_{i=1}^{N} \kappa_{i}}{N}
$$

is shown in the graphs, where $N$ is the number of constellations (e.g., $N=128$ ) and $\kappa_{i}$ is the logarithmic antenna condition number of a constellation $i$. 


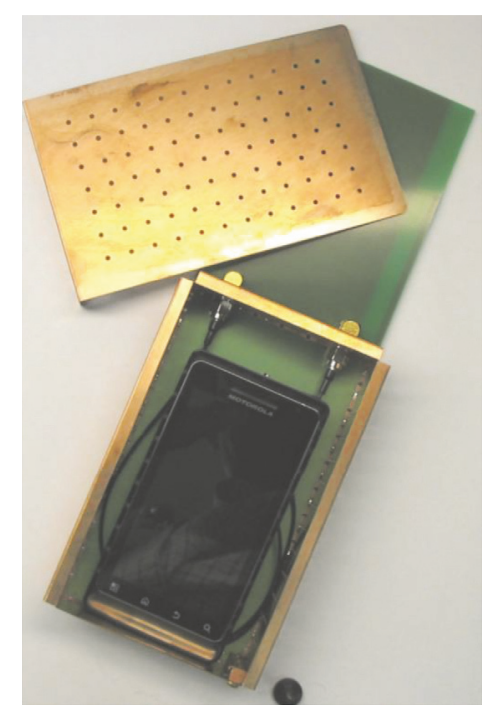

FIGURE 17: LTE reference antenna for UE with two antennas designed by Motorola Mobility for band $13(751 \mathrm{MHz})$, with a printed circuit board carrying a closed metal cavity for the UE, two printed antennas, and connection cables [28].
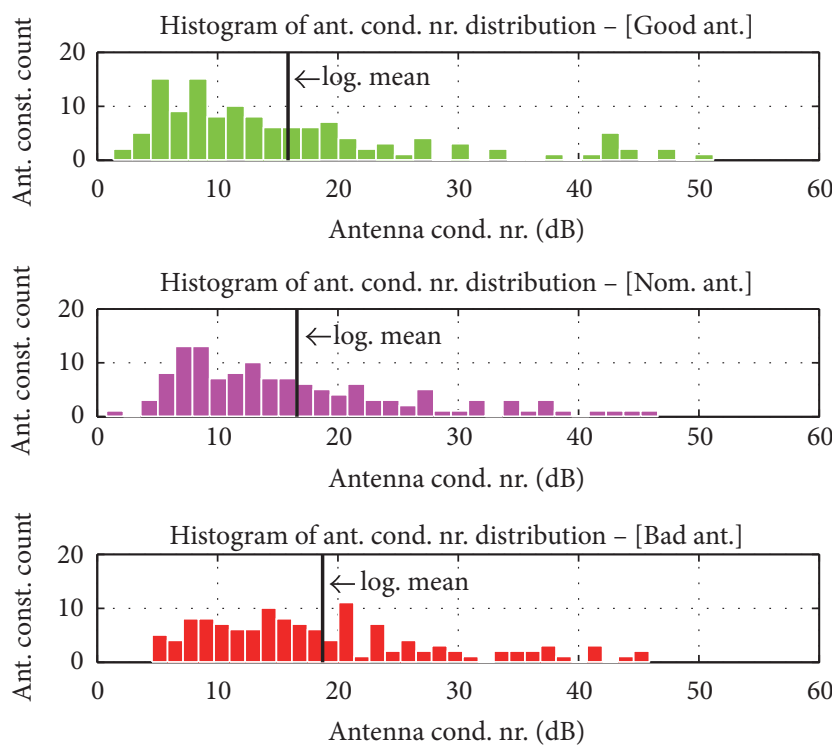

FIGURE 18: Distribution of the antenna condition number and mean logarithmic condition number of the "Good"; "Nominal"; and "Bad" reference antennas.

The reference antennas behave as they are named. Going from "Good" to "Bad", $\bar{\kappa}$ rises:

(i) “Good" antenna: $15.86 \mathrm{~dB}$

(ii) "Nominal" antenna: $16.6 \mathrm{~dB}$

(iii) "Bad" antenna: $18.72 \mathrm{~dB}$

Table 2 summarizes the mean logarithmic deviations $\bar{d}$ for the same parameter variation as in Section 5.4.2 for a relative throughput of $70 \%$ :

(i) DLSCH processing on/off (ii) Ideal/nonideal channel estimation

(iii) Low, medium, and high channel correlations

The mean logarithmic deviation is

$$
\bar{d}=\frac{\sum_{i=1}^{N} d_{i}}{N},
$$

where $N$ again is the total number of constellations and $d_{i}$ is the deviation at the constellation $i$. The DM can be validated for all three types of antennas within a $|\bar{d}|$ of $4.69 \mathrm{~dB}$. This maximum of the averaged values of $\bar{d}$ lies below the peak value of $|d|$, as shown in Figure 15. In the case UE employs LTE, DLSCH processing is always applied. In this case the absolute mean logarithmic deviation $|\bar{d}|$ will always be below $3.57 \mathrm{~dB}$ for all antenna condition numbers. If LTE UE is tested only with low correlation of channels, the method works almost perfectly with $|\bar{d}|$ below $0.29 \mathrm{~dB}$.

Finally, the following can be pointed out:

(i) The mean deviation $|\bar{d}|$ is always below the maximum of the absolute deviations $|d|$. In the case of ICD/ZF receiver, low correlated channel, DLSCH processing, and nonideal channel measurement even for the "Bad" antenna it will be only $0.1 \mathrm{~dB}$.

(ii) In the practical case of rather well conditioned antenna matrices and DLSCH processing applied, the method works very well.

(iii) Comparing the receiver types, the MMSE receiver causes higher mean deviations $\bar{d}$ than the ICD/ZF receiver.

(iv) The investigations exhibit that the mean deviation $\bar{d}$ decreases when going from antenna type "Bad" to antenna type "Good."

(v) If a very poor antenna system is measured, there is a possibility of calibrating the system, as the deviation $d$ is static.

(vi) The DM is an innovative method to deliver fast and reliable performance test results.

\section{Validation of the DM by OTA Measurements of UE}

6.1. Measurements. The authors conducted measurements with a commercial LTE device, namely, the Sony Xperia smartphone, for a round-robin campaign for radio area network group 4 (RAN4) of 3GPP. They tested the UE in an anechoic chamber using the "Good" antenna, employing the setup in Figure 2 at 128 constellations [30]. The authors measured the "DM" results and the "overall" results of the Sony Xperia smartphone with different transmission channel models than in the simulation to calculate the deviation $d$.

Figure 19 shows the experimental results of the distribution of the deviation $d$. The authors determined the deviation $d$ for the different transmission channel models for all 128 constellations. The results of $d$ for the different channel models are color coded in the diagram. 
TABLE 2: Mean logarithmic deviations $\bar{d}$ at 70\% relative throughput using LTE reference antennas "Good"; "Nominal"; and "Bad" with parameters: channel correlation type, DLSCH processing, and channel estimation.

\begin{tabular}{|c|c|c|c|c|c|c|}
\hline \multirow{2}{*}{ Receiver type } & \multirow{2}{*}{ Corr. } & \multirow{2}{*}{ DLSCH proc. } & \multirow{2}{*}{ ch. est. } & \multicolumn{3}{|c|}{ Deviation $\bar{d}$ for ref. ant./dB } \\
\hline & & & & "Good" & "Nominal" & "Bad" \\
\hline $\mathrm{ICD} / \mathrm{ZF}$ & Low & Off & Ideal & -0.26 & -0.26 & -0.29 \\
\hline $\mathrm{ICD} / \mathrm{ZF}$ & Low & Off & Nonideal & -0.19 & -0.23 & -0.26 \\
\hline $\mathrm{ICD} / \mathrm{ZF}$ & Low & On & Ideal & 0.12 & 0.13 & 0.13 \\
\hline $\mathrm{ICD} / \mathrm{ZF}$ & Low & On & Nonideal & 0.09 & 0.10 & 0.10 \\
\hline $\mathrm{ICD} / \mathrm{ZF}$ & Med & Off & Ideal & -2.73 & -2.86 & -3.02 \\
\hline $\mathrm{ICD} / \mathrm{ZF}$ & Med & Off & Nonideal & -2.71 & -2.86 & -3.02 \\
\hline $\mathrm{ICD} / \mathrm{ZF}$ & Med & On & Ideal & -2.37 & -2.47 & -2.59 \\
\hline $\mathrm{ICD} / \mathrm{ZF}$ & Med & On & Nonideal & -2.38 & -2.47 & -2.58 \\
\hline $\mathrm{ICD} / \mathrm{ZF}$ & High & Off & Ideal & -2.67 & -2.81 & -2.96 \\
\hline $\mathrm{ICD} / \mathrm{ZF}$ & High & Off & Nonideal & -2.69 & -2.82 & -2.98 \\
\hline $\mathrm{ICD} / \mathrm{ZF}$ & High & On & Ideal & -2.37 & -2.47 & -2.59 \\
\hline $\mathrm{ICD} / \mathrm{ZF}$ & High & On & Nonideal & -2.36 & -2.47 & -2.59 \\
\hline MMSE & Low & Off & Ideal & -1.39 & -1.49 & -1.65 \\
\hline MMSE & Low & Off & Nonideal & -1.39 & -1.52 & -1.77 \\
\hline MMSE & Low & On & Ideal & -0.61 & -0.64 & -0.71 \\
\hline MMSE & Low & On & Nonideal & -0.53 & -0.56 & -0.66 \\
\hline MMSE & Med & Off & Ideal & -3.82 & -4.14 & -4.46 \\
\hline MMSE & Med & Off & Nonideal & -3.90 & -4.14 & -4.51 \\
\hline MMSE & Med & On & Ideal & -3.13 & -3.26 & -3.43 \\
\hline MMSE & Med & On & Nonideal & -3.18 & -3.37 & -3.58 \\
\hline MMSE & High & Off & Ideal & -4.00 & -4.27 & -4.63 \\
\hline MMSE & High & Off & Nonideal & -3.9 & -4.14 & -4.49 \\
\hline MMSE & High & On & Ideal & -3.2 & -3.36 & -3.57 \\
\hline MMSE & High & On & Nonideal & -3.17 & -3.36 & -3.55 \\
\hline
\end{tabular}

The measurement setup employs a full LTE transmission containing tapped delay channel models, OFDM, and resource block allocation [7]. These tapped delay models were used: Urban Micro (Umi), Urban Micro (Umi) with all tap correlations $\alpha=0$, and Urban Macro (Uma). These models are proposed for OTA testing of UE by 3rd-Generation Partnership Project (3GPP) [43].

In comparison to the measurements, the simulations in Section 5 use a simplified setup. Nevertheless, a comparison is valid, as the DM is validated by simulation for a single OFDM subcarrier that has a bandwidth of $15 \mathrm{kHz}$, in the LTE case. This narrow bandwidth allows a treatment of the channel as frequency flat-fading ones. The DM is validated for the smallest element, the single OFDM channel. Therefore it is also valid for the complete fading channel, which consists of many small OFDM carriers.

Figure 19 points out that the deviations $d$ between the measured "overall" and DM results are distributed around mean values from $-0.16 \mathrm{~dB}$ to $0.34 \mathrm{~dB}$ for all employed channel models, which is very low regarding all other uncertainty sources $[41,42]$. The approximately Gaussian shaped distribution of the deviation $d$ is resulting from the mentioned measurement uncertainties.
6.2. Comparison of Measurements to the Simulations. The measurements in Section 6.1 were conducted with a slightly different set of parameters as proposed by 3GPP $[33,35]$. The authors present another set of simulations, as close as possible to the measurements. The channel models employ tapped delay models, and they were used in the measurements. The taps have different correlation values $\alpha$, while $\beta$ is always zero. To be able to use the different correlations of the taps for the simplified model, the authors employ a linear power related averaging of $\alpha$. This averaging results in these values of $\alpha$ :

(i) Channel model Uma: $\alpha=0.1172$ (light correlation).

(ii) Channel model Umi with $(\alpha=0)$ : the mean value also is $\alpha=0$ (all taps are zero; uncorrelated).

(iii) Channel model Umi: $\alpha=0.9474$ (strong correlation).

Results are summarized in Table 3. The mean deviation $\bar{d}$ is given for the measurements and the closest possible simulations for several channel models. The mean deviation $\bar{d}$ is very close to zero. So to say, all validations with theoretical results, simulation results, and measurement results (Table 2) point in the same direction: the validity of the DM. The mean value of the deviation $\bar{d}$ is within the given limits for all employed channel models. 


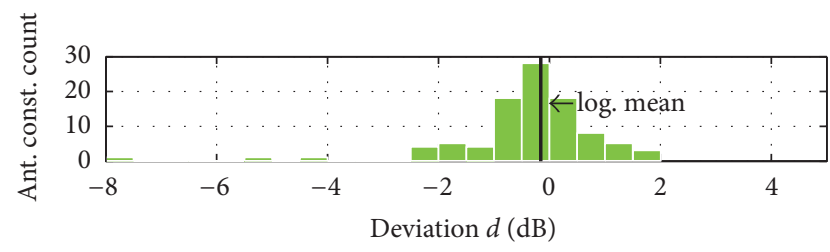

(a) Channel model: Uma

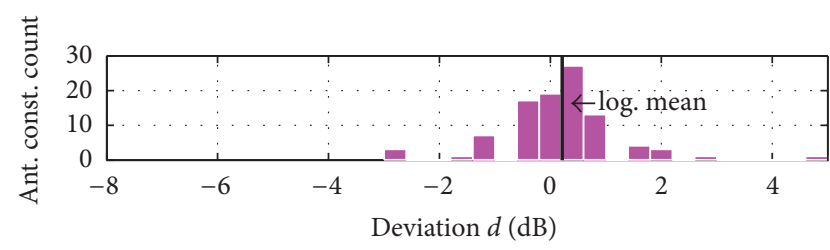

(b) Channel model: Umi

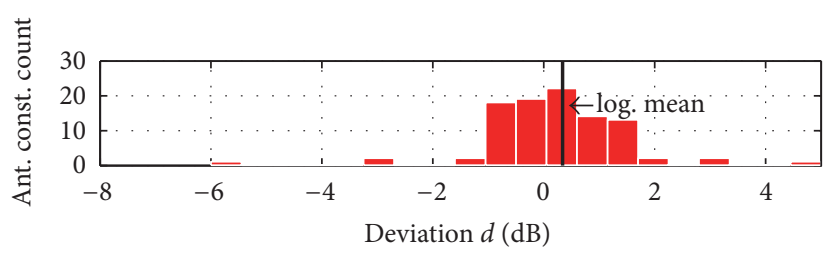

(c) Channel model: Umi $\alpha=0$

FIGURE 19: Distribution of the log. deviations $d$ between the DM and "overall” measurement results for different channel models (Uma; Umi; Umi_alpha $=0$ ) and different base-station correlation coefficient for the smartphone Sony Xperia as UE with "Good" reference antenna.

TABLE 3: Comparison of the deviation $\bar{d}$ between measurement and simulations.

\begin{tabular}{lccc}
\hline Channel model & & Deviation $\bar{d}$ in $\mathrm{dB}$ for \\
& Simulation ICD/ZF & Simulation MMSE \\
\hline Uma & -0.16 & 0.1 & -0.57 \\
Umi & 0.21 & 0.05 & -0.43 \\
Umi $\alpha=0$ & 0.34 & -0.42 & -0.52 \\
\hline
\end{tabular}

\section{Conclusion}

This paper covers performance testing of Multiple-Input Multiple-Output (MIMO) enabled wireless user equipment. It deals with over-the-air (OTA) performance tests for complete MIMO enabled user equipment (UE), including antennas and front-end and algorithmic performance. The authors investigate a very promising method, the Decomposition Method (DM). It does not compare the DM to other test procedures.

The best way to test UE would be to check the algorithmic and the antenna performance at the same time. Unfortunately, this is very slow. To speed up the measurement procedure, the Decomposition Method splits the performance tests into two parts: a static test for the antenna performance and a fading test for the algorithmic performance. The results from the separated measurements are combined and deliver accurate and repeatable results. By applying this split, the measurements can be speeded up by a factor of 36 in comparison to the combined measurements.

This paper introduces the Decomposition Method and deals with the validation of the split of the measurements. The authors validate the Decomposition Method by theoretical investigations and by numerical simulation as well as by measurement.

As many MIMO enabled devices also use Orthogonal Frequency Division Multiplexing (OFDM), the single orthogonal frequency bands have small bandwidths. The paper exploits this fact by simplifying the employed models from generic tapped delay channel models to frequency flat response channels, without loosing validity.

The authors validate the method by theoretical means using the MIMO channel capacity. They also validate it by numerical means, simulating a complete transmission system with Kronecker channel models with frequency flat fading. They show that the applicability of the Decomposition Method is given for most use cases. Low correlated channels deliver an absolute deviation of the Decomposition Method result lower than $0.7 \mathrm{~dB}$.

To round up the picture, the validity of the method is shown by measurement results for the Sony Xperia smartphone. The mean deviation $\bar{d}$ is within the interval $\{-0.16 \mathrm{~dB}$ to $0.34 \mathrm{~dB}$.

The authors provide a thorough scientific investigation of the Decomposition Method, demonstrating that this powerful and quick method to rate the performance of a MIMO enabled wireless UE is valid in most use cases. The method is able to speed up tests and still delivers accurate and reliable results with reasonable equipment effort.

\section{Conflicts of Interest}

The authors declare that they have no conflicts of interest.

\section{References}

[1] Third Generation Partnership Project (3GPP), Universal Terrestrial Radio Access (UTRA) and Evolved Universal Terrestrial Radio Access; Verification of Radiated Multi-Antenna Reception 
Performance of User Equipment (UE), 2014.

[2] M. D. Foegelle, "Creating a complex multipath environment simulation in an anechoic chamber," Microwave Journal, vol. 53, no. 8, pp. 56-64, 2010.

[3] M. Rumney, LTE and the Evolution to $4 G$ Wireless-Design and Measurement Challenges, John Wiley \& Sons, New York, NY, USA, 2013.

[4] I. Cartón Llorente, W. Fan, and G. F. Pedersen, "Mimo ota testing in small multiprobe anechoic chamber setups," IEEE Antennas and Wireless Propagation Letters, vol. 15, pp. 1167-1170, 2016.

[5] W. Fan, X. Carreño, P. Kyösti, J. Ø. Nielsen, and G. F. Pedersen, "Over-the-air testing of MIMO-capable terminals: evaluation of multiple-antenna systems in realistic multipath propagation environments using an OTA method," IEEE Vehicular Technology Magazine, vol. 10, no. 2, pp. 38-46, 2015.

[6] M. D. Foegelle, "The future of MIMO over-the-air testing," IEEE Communications Magazine, vol. 52, no. 9, pp. 134-142, 2014.

[7] P.-S. Kildal, C. Orlenius, and J. Carlsson, "OTA testing in multipath of antennas and wireless devices with MIMO and OFDM," Proceedings of the IEEE, vol. 100, no. 7, pp. 2145-2157, 2012.

[8] W. Fan, P. Kyösti, L. Hentilä, J. Ø. Nielsen, and G. F. Pedersen, "Rician channel modeling for multiprobe anechoic chamber setups," IEEE Antennas and Wireless Propagation Letters, vol. 13, pp. 1761-1764, 2014.

[9] W. Fan, F. Sun, J. Ø. Nielsen et al., "Probe selection in multiprobe OTA setups," IEEE Transactions on Antennas and Propagation, vol. 62, no. 4, pp. 2109-2120, 2014.

[10] Q. Xu, Y. Huang, X. Zhu, S. S. Alja'afreh, and L. Xing, “A new antenna diversity gain measurement method using a reverberation chamber," IEEE Antennas and Wireless Propagation Letters, vol. 14, pp. 935-938, 2015.

[11] W. Yu, Y. Qi, K. Liu, Y. Xu, and J. Fan, "Radiated two-stage method for LTE MIMO user equipment performance evaluation," IEEE Transactions on Electromagnetic Compatibility, vol. 56, no. 6, pp. 1691-1696, 2014.

[12] W. Fan, X. Carren, J. O. Nielsen, K. Olesen, M. B. Knudsen, and G. F. Pedersen, "Measurement verification of plane wave synthesis technique based on multiprobe MIMO-OTA setup," in Proceedings of the IEEE Vehicular Technology Conference (VTC Fall '12), pp. 1-5, September 2012.

[13] A. Khatun, V.-M. Kolmonen, T. Laitinen, and K. Nikoskinen, "Clarification of uncertainties in MIMO over-the-air multiprobe test systems," in Proceedings of the 7th European Conference on Antennas and Propagation (EuCAP '13), pp. 1427-1431, April 2013.

[14] W. Fan, J. O. Nielsen, X. Carreno, J. S. Ashta, G. F. Pedersen, and M. B. Knudsen, "Impact of system non-idealities on spatial correlation emulation in a multi-probe based MIMO OTA setup," in Proceedings of the 7th European Conference on Antennas and Propagation (EuCAP '13), pp. 1663-1667, April 2013.

[15] A. Scannavini, L. J. Foged, M. Anouar, N. Gross, and J. Estrada, "OTA throughput measurements by using spatial fading emulation technique," in Proceedings of 4th European Conference on Antennas and Propagation (EuCAP '10), pp. 1-5, April 2010.

[16] S.-S. Oh, J.-M. Kim, J.-H. Yun, and S.-Y. Kang, "Antenna measurement method in fresnel region by $\varphi$-variation scanning," IEEE Antennas and Wireless Propagation Letters, vol. 7, pp. 206209, 2008.
[17] A. Adardour, G. Andrieu, and A. Reineix, "Influence of a stirrer on the cavity modes within a reverberation chamber," in Proceedings of the International Symposium on Electromagnetic Compatibility (EMC EUROPE '12), pp. 1-4, September 2012.

[18] J. F. Dawson and L. Arnaut, "Reverberation (modestirred) chambers for electromagnetic compatibility," June 2007, http:// www.compliance-club.com/archive/old_archive/030530.htm.

[19] P. Plaza-González, J. Monzó-Cabrera, J. M. Catalá-Civera, and D. Sánchez-Hernández, "New approach for the prediction of the electric field distribution in multimode microwave-heating applicators with mode stirrers," IEEE Transactions on Magnetics, vol. 40, no. 3, pp. 1672-1678, 2004.

[20] B. Zhang, Z. Yuan, and J. He, "Comparison on the test results between reverberation chamber and anechoic chamber," in Proceedings of the Asia-Pacific Symposium on Electromagnetic Compatibility (APEMC '12), pp. 769-772, IEEE, Singapore, May 2012.

[21] C. Bruns and R. Vahldieck, "A closer look at reverberation chambers-3-D simulation and experimental verification," IEEE Transactions on Electromagnetic Compatibility, vol. 47, no. 3, pp. 612-626, 2005.

[22] A. B. Ubin and M. Z. B. M. Jenu, "Field uniformity evaluation of different stirrer structure in a reverberation chamber," in Proceedings of the 5th IEEE Asia-Pacific Conference on Applied Electromagnetics (APACE '12), pp. 32-35, December 2012.

[23] B. Auinger, On the performance testing of MIMO-enabled wireless mobile user devices [Ph.D. thesis], Graz University of Technology, Graz, Austria, 2015.

[24] C. von Gagern, "Decomposition test results from the second CTIA round robin test," COST IC1004, (TD(13)07049), 2013.

[25] B. Auinger, M. Gadringer, A. Tankielun, C. V. Gagern, and W. Boesch, "Numerical analysis of the decomposition method using LTE reference antennas," in Proceedings of the International Conference on Computer and Network Engineering (ICCNE '14), December 2014.

[26] A. Tankielun, "Two-Channel Method for OTA performance measurements of MIMO-enabled devices," White Paper, Rohde \& Schwarz, Munich, Germany, 2011.

[27] H. Zarrinkoub, Understanding LTE with MATLAB- From Mathematical Modelling to Simulation and Prototyping, Wiley, West Sussex, UK, 1st edition, 2014.

[28] I. Szini, "Reference antennas proposal for MIMO OTA," European Cooperation in Science and Technology (COST) IC1004, Document Nr. 1(TD(11)02009), October 2011.

[29] C. Gagern and A. Tankielun, "Test results from the second CTIA round robin test," The Wireless Association (CTIA), February 2013.

[30] A. Tankielun and C. Von Gagern, "On spatial characteristics of the decomposition method in MIMO OTA testing," in Proceedings of the 8th European Conference on Antennas and Propagation (EuCAP '14), pp. 3674-3678, Hague, The Netherlands, April 2014.

[31] A. Paulraj, R. Nabar, and D. Gore, Introduction to Space-Time Wireless Communications, Cambridge University Press, Cambridge, UK, 2003.

[32] D. Kececioglu, Reliability Engineering Handbook Volume 1, DEStech Publications, Lancaster, Pa, USA, 2002.

[33] 3GPP, “Technical specification group radio access network; evolved universal terrestrial radio access (EUTRA); user equipment (UE) radio transmission and reception," (Release 12), September 2014. 
[34] C. Oestges, "Validity of the Kronecker model for MIMO correlated channels," in Proceedings of the IEEE 63rd Vehicular Technology Conference (VTC '06), vol. 6, pp. 2818-2822, Melbourne, Australia, July 2006.

[35] ETSI AND 3GPP, Technical Specification: LTE; Evolved Universal Terrestrial Radio Access (E-UTRA); Base Station (BS) Conformance Testing (3GPP TS 36.141 Version 12.5.0 Release 12), 2014.

[36] U. Mengali, Synchronization Techniques for Digital Receivers, Plenum Press, New York, NY, USA, 1997.

[37] J. G. Proakis, Digital Communications, McGraw-Hill, New York, NY, USA, 5th edition, 2008.

[38] N. J. Salkind, Encyclopedia of Research Design, Sage, Thousand Oaks, Calif, USA, 1st edition, 2010.

[39] E. Telatar, "On limits of wireless communications in a fading environment when using multiple antennas," European Transactions on Telecommunications, vol. 10, no. 6, pp. 585-595, 1999.

[40] W. Fan, I. Szini, M. D. Foegelle, J. O. Nielsen, and G. F. Pedersen, "Measurement uncertainty investigation in the multi-probe OTA setups," in Proceedings of the 8th European Conference on Antennas and Propagation (EuCAP '14), pp. 1068-1072, Hague, The Netherlands, April 2014.

[41] Third Generation Partnership Project (3GPP), "Errors and uncertainties in the mimo ota measurements r4-131673," Meeting 65, New Orleans, November 2012.

[42] 3GPP, "Measurement uncertainty evaluation of multiprobe method," in Proceedings of the 3GPP Meeting 66, Chicago, Ill, USA, November 2012

[43] CTIA, "Draft CTIA MIMO OTA test plan version 0.2 (CTIA MOSG131214; sub working group document available to CTIA sub-working group members)," December 2013. 


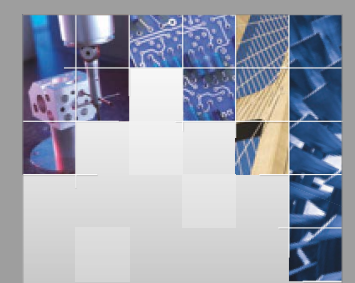

\section{Enfincering}
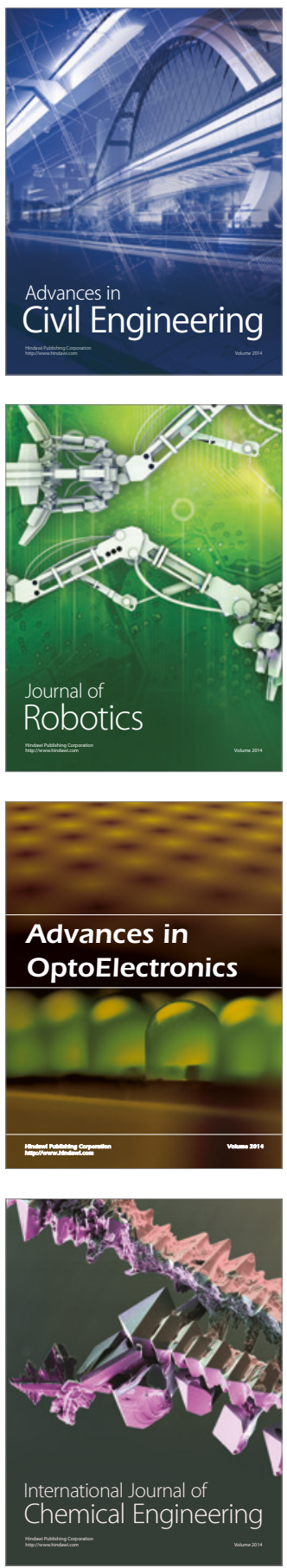

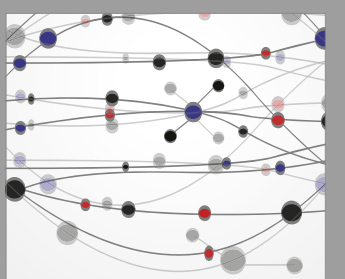

The Scientific World Journal

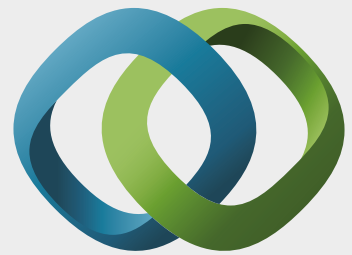

\section{Hindawi}

Submit your manuscripts at

https://www.hindawi.com
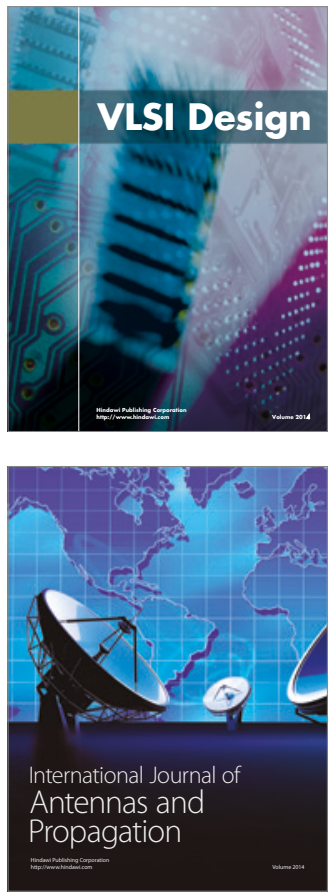

\section{Rotating}

Machinery
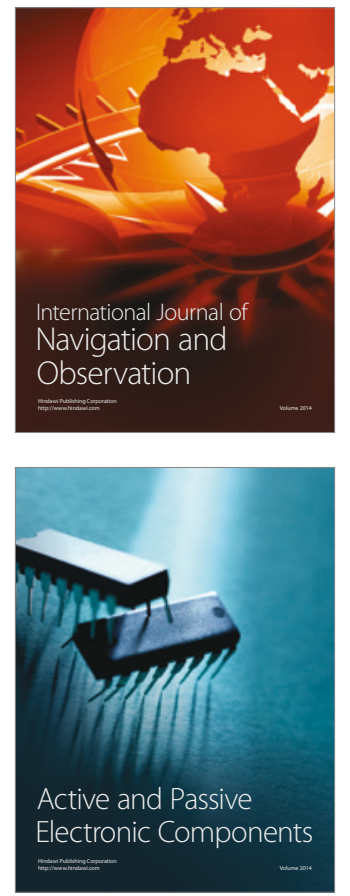
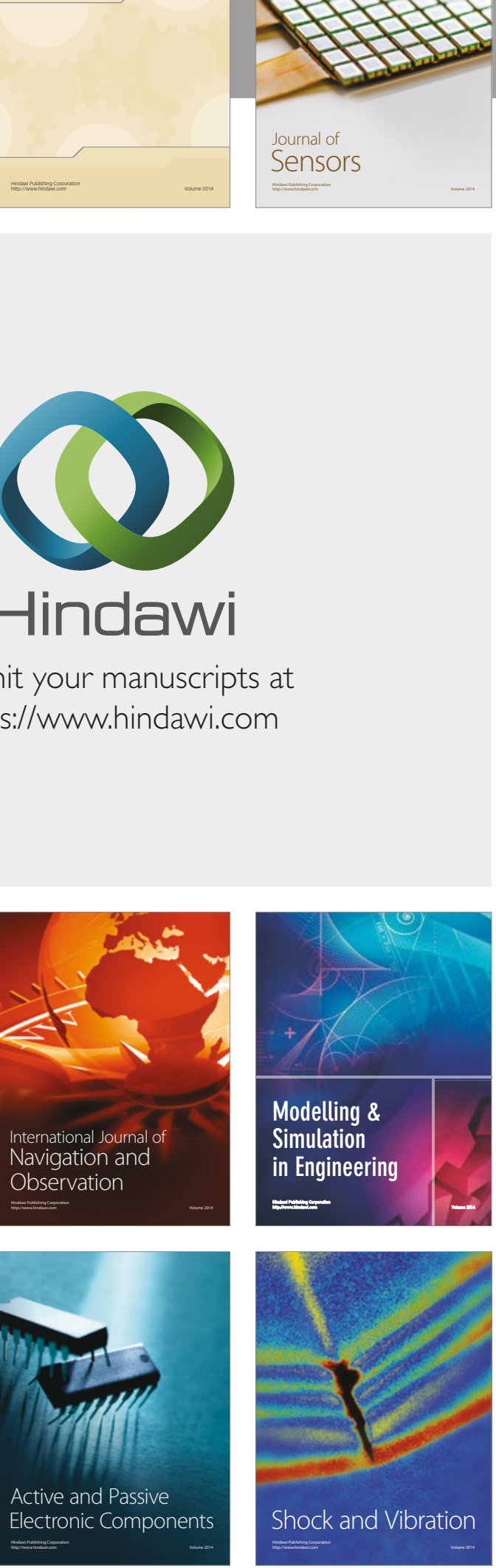
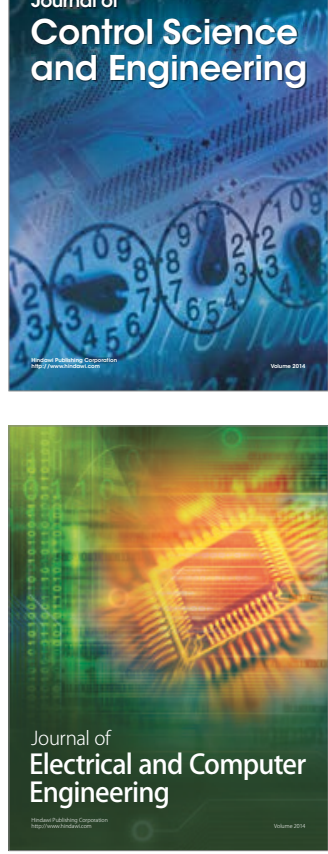

Distributed

Journal of

Control Science

and Engineering
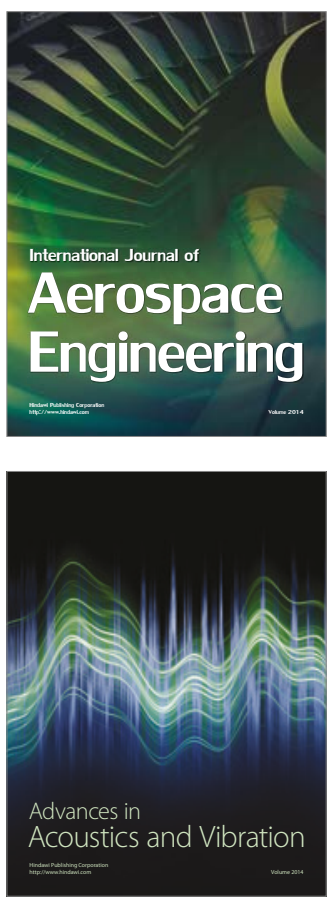

Sensor Networks 